\title{
MULTIPLICITY OF SOLUTIONS FOR RESONANT NEUMANN PROBLEMS WITH AN INDEFINITE AND UNBOUNDED POTENTIAL
}

\author{
NIKOLAOS S. PAPAGEORGIOU AND VICENŢIU D. RĂDULESCU
}

\begin{abstract}
We examine semilinear Neumann problems driven by the Laplacian plus an unbounded and indefinite potential. The reaction is a Carathéodory function which exhibits linear growth near $\pm \infty$. We allow for resonance to occur with respect to a nonprincipal nonnegative eigenvalue, and we prove several multiplicity results. Our approach uses critical point theory, Morse theory and the reduction method (the Lyapunov-Schmidt method).
\end{abstract}

\section{INTRODUCTION}

Let $\Omega \subseteq \mathbb{R}^{N}$ be a bounded domain with a $C^{2}$-boundary $\partial \Omega$. In this paper, we study the following semilinear Neumann problem:

$$
-\Delta u(z)+\beta(z) u(z)=f(z, u(z)) \text { in } \Omega, \frac{\partial u}{\partial n}=0 \text { on } \partial \Omega .
$$

Here $n(\cdot)$ denotes the outward unit normal on $\partial \Omega$. The potential function $\beta(\cdot)$ is in general unbounded and sign changing. More precisely, $\beta \in L^{s}(\Omega)$ with $s>$ $N$. Also, the reaction $f(z, x)$ is a Carathéodory function (that is, for all $x \in$ $\mathbb{R}, z \mapsto f(z, x)$ is measurable and for a.a. $z \in \Omega, x \mapsto f(z, x)$ is continuous), which exhibits linear growth near $\pm \infty$. We allow for resonance to occur with respect to any nonnegative nonprincipal eigenvalue of $\left(-\Delta+\beta(\cdot), H^{1}(\Omega)\right)$. So, we assume that asymptotically at $\pm \infty$ the quotient $\frac{f(z, x)}{x}$ is located in the spectral interval $\left[\hat{\lambda}_{m}, \hat{\lambda}_{m+1}\right]$ with $m \geqslant \max \left\{m_{0}, 2\right\}$, where $\hat{\lambda}_{m_{0}}$ is the first nonnegative eigenvalue of $\left(-\Delta+\beta(\cdot), H^{1}(\Omega)\right)$. Hence, if $\beta \equiv 0$, then $m_{0}=2$ and so $m \geqslant 2$. We allow resonance with respect to the left end $\hat{\lambda}_{m}$ and nonuniform nonresonance with respect to the right end $\hat{\lambda}_{m+1}$. Problems with double resonance (that is, possible resonance at both ends of the spectral interval) were studied by O'Regan, Papageorgiou \& Smyrlis [25, with $\beta \equiv 0$ (see also Hu \& Papageorgiou [16] for Dirichlet problems with $\beta \neq 0$ ). After that, we deal with equations which are resonant at the origin and their energy functional is indefinite. Our aim is to prove multiplicity theorems for these problems. In the past analogous multiplicity results were proved for Dirichlet problems with $\beta \equiv 0$ by Bartsch \& Wang [4, Castro, Cossio \& Velez [6, Castro \& Lazer [7, Hofer [15], Liu [18, Liu \& Li [20] and Thews 32]. Dirichlet problems with an unbounded, indefinite potential were studied recently by Gasinski \& Papageorgiou [12, Kyritsi \& Papageorgiou [17,

Received by the editors December 20, 2013.

2010 Mathematics Subject Classification. Primary 35J20, 35J60, 58 E05.

Key words and phrases. Indefinite and unbounded potential, reduction method, resonance, unique continuation property, regularity, critical groups. 
Papageorgiou \& Papalini [26]. Resonant Neumann problems were investigated by Filippakis \& Papageorgiou [10, Gasinski \& Papageorgiou [13, Motreanu, Motreanu \& Papageorgiou [22, Tang [30] and Tang \& Wu [31]. Neumann equations with unbounded, indefinite potential were studied by Papageorgiou \& Rădulescu [27] (problems with crossing nonlinearity) and Papageorgiou \& Smyrlis 28] (coercive problems).

Our approach uses variational methods based on the critical point theory, coupled with the reduction technique (the Lyapunov-Schmidt method) and with Morse theory (critical groups). In the next section, for convenience of the reader, we review the main mathematical tools which we will use in the sequel.

\section{Mathematical BACKGround}

Let $X$ be a Banach space and $X^{*}$ its topological dual. By $\langle\cdot, \cdot\rangle$ we denote the duality brackets for the pair $\left(X^{*}, X\right)$. Let $\varphi \in C^{1}(X)$. We say that $\varphi$ satisfies the " $C$-condition", if the following holds:

"Every sequence $\left\{u_{n}\right\}_{n \geqslant 1} \subseteq X$ such that $\left\{\varphi\left(u_{n}\right)\right\}_{n \geqslant 1} \subseteq \mathbb{R}$ is bounded and

$$
\left(1+\left\|u_{n}\right\|\right) \varphi^{\prime}\left(u_{n}\right) \rightarrow 0 \text { in } X^{*} \text { as } n \rightarrow \infty,
$$

admits a strongly convergent subsequence".

This compactness-type condition on the functional $\varphi$ is more general than the better known Palais-Smale condition. Nevertheless, the $C$-condition suffices to prove a deformation theorem and from it derive the minimax theory of certain critical values of $\varphi$. One such minimax theorem, which we will use later, is the so-called "mountain pass theorem".

Theorem 1. Assume that $X$ is a Banach space, $\varphi \in C^{1}(X)$ satisfies the $C$ condition, $u_{0}, u_{1} \in X$, with $\left\|u_{1}-u_{0}\right\|>\varrho>0$,

$$
\begin{aligned}
& \max \left\{\varphi\left(x_{0}\right), \varphi\left(x_{1}\right)\right\}<\inf \left[\varphi(u):\left\|u-u_{0}\right\|=\varrho\right]=\eta_{\varrho} \\
& \text { and } \quad c=\inf _{\gamma \in \Gamma} \max _{0 \leqslant t \leqslant 1} \varphi(\gamma(t)) \text { with } \Gamma=\left\{\gamma \in C([0,1], X): \gamma(0)=u_{0}, \gamma(1)=u_{1}\right\} .
\end{aligned}
$$

Then $c \geqslant \eta_{\varrho}$ and $c$ is a critical value of $\varphi$.

In the analysis of problem (1), in addition to the Sobolev space $H^{1}(\Omega)$, we will also use the Banach space $C^{1}(\bar{\Omega})$. This is an ordered Banach space with positive cone

$$
C_{+}=\left\{u \in C^{1}(\bar{\Omega}): u(z) \geqslant 0 \text { for all } z \in \bar{\Omega}\right\} .
$$

This cone has a nonempty interior given by

$$
\operatorname{int} C_{+}=\left\{u \in C_{+}: u(z)>0 \text { for all } z \in \bar{\Omega}\right\} .
$$

For a Banach space $(X,\|\cdot\|)$, we say that it has the Kadec-Klee property if the following is true (see Gasinski \& Papageorgiou [11, p. 911]):

$$
\text { " } u_{n} \stackrel{w}{\longrightarrow} u \text { in } X \text { and }\left\|u_{n}\right\| \rightarrow\|u\| \text { imply } u_{n} \rightarrow u \text { in } X " .
$$

It is well known that locally uniformly convex Banach spaces (in particular, Hilbert spaces) have the Kadec-Klee property.

In what follows, by $\|\cdot\|$ we denote the norm of $H^{1}(\Omega)$. Hence

$$
\|u\|=\left[\|u\|_{2}^{2}+\|D u\|_{2}^{2}\right]^{1 / 2} \text { for all } u \in H^{1}(\Omega)
$$


(here by $\|\cdot\|_{2}$ we denote the norm of $L^{2}(\Omega)$ and $L^{2}\left(\Omega, \mathbb{R}^{N}\right)$ ). Also, for every $x \in \mathbb{R}$, we set $x^{ \pm}=\max \{0, \pm x\}$. Then for every $u \in H^{1}(\Omega)$ we define $u^{ \pm}(\cdot)=u(\cdot)^{ \pm}$. We know that

$$
u^{ \pm} \in H^{1}(\Omega), u=u^{+}-u^{-} \text {and }|u|=u^{+}+u^{-} \text {for all } u \in H^{1}(\Omega) .
$$

By $|\cdot|_{N}$ we denote the Lebesgue measure on $\mathbb{R}^{N}$. Finally, if $h: \Omega \times \mathbb{R} \rightarrow \mathbb{R}$ is a measurable function (for example, a Carathéodory function), then we set

$$
N_{h}(u)(\cdot)=h(\cdot, u(\cdot)) \text { for all } u \in H^{1}(\Omega) .
$$

The following linear eigenvalue problem has a pivotal role in the analysis of problem (11):

$$
-\Delta u(z)+\beta(z) u(z)=\lambda u(z) \text { in } \Omega, \frac{\partial u}{\partial n}=0 \text { on } \partial \Omega .
$$

This eigenvalue problem was studied by Papageorgiou \& Smyrlis [28]. So, suppose that $\beta \in L^{N / 2}(\Omega)$ if $N \geqslant 3, \beta \in L^{r}(\Omega)$ with $r>1$ if $N=2$ and $\beta \in L^{1}(\Omega)$ if $N=1$. Let $\tau: H^{1}(\Omega) \rightarrow \mathbb{R}$ be the functional defined by

$$
\tau(u)=\|D u\|_{2}^{2}+\int_{\Omega} \beta(z) u(z)^{2} d z \text { for all } u \in H^{1}(\Omega) .
$$

Then the eigenvalue problem (2) has a smallest eigenvalue $\hat{\lambda}_{1}>-\infty$ given by

$$
\hat{\lambda}_{1}=\inf \left[\frac{\tau(u)}{\|u\|_{2}^{2}}: u \in H^{1}(\Omega), u \neq 0\right] .
$$

From (3) it follows that we can find $\xi_{0}>\max \left\{-\hat{\lambda}_{1}, 0\right\}$ such that

$$
\tau(u)+\xi_{0}\|u\|_{2}^{2} \geqslant c_{1}\|u\|^{2} \text { for all } u \in H^{1}(\Omega) \text { and some } c_{1}>0 .
$$

Using (41) and the spectral theorem for compact self-adjoint operators (see, for example, Gasinski \& Papageorgiou [11, p. 297]), we obtain a sequence $\left\{\hat{\lambda}_{k}\right\}_{k \geqslant 1}$ consisting of all the eigenvalues of (2) such that $\hat{\lambda}_{k} \rightarrow+\infty$ when $k \rightarrow \infty$. To these eigenvalues corresponds a sequence $\left\{\hat{u}_{n}\right\}_{n \geqslant 1} \subseteq H^{1}(\Omega)$ of eigenfunctions which form an othonormal basis of $L^{2}(\Omega)$ and an orthogonal basis of $H^{1}(\Omega)$. Moreover, if $\beta \in L^{s}(\Omega)$ with $s>N$, the the regularity results of Wang 34] imply that $\left\{\hat{u}_{n}\right\}_{n \geqslant 1} \subseteq C^{1}(\bar{\Omega})$. These eigenvalues admit variational characterizations in terms of the Rayleigh quotient $\frac{\tau(u)}{\|u\|_{2}^{2}}$ for all $u \in H^{1}(\Omega) \backslash\{0\}$. In what follows, by $E\left(\hat{\lambda}_{k}\right)$ we denote the eigenspace corresponding to the eigenvalue $\hat{\lambda}_{k}, k \geqslant 1$. For $\hat{\lambda}_{1}$ the variational characterization is given by (3) and the infimum is in fact a minimum which is realized on $E\left(\hat{\lambda}_{1}\right)$. For the higher eigenvalues, we have:

$$
\begin{aligned}
\hat{\lambda}_{i} & =\inf \left[\frac{\tau(u)}{\|u\|_{2}^{2}}: u \in \overline{\bigoplus_{k \geqslant i} E\left(\lambda_{k}\right)}, u \neq 0\right] \\
& =\sup \left[\frac{\tau(u)}{\|u\|_{2}^{2}}: u \in \bigoplus_{k=1}^{i} E\left(\hat{\lambda}_{i}\right), u \neq 0\right] \text { for all } i \geqslant 2 .
\end{aligned}
$$

The infimum and the supremum in (5) are realized on $E\left(\hat{\lambda}_{i}\right), i \geqslant 2$. The first eigenvalue $\hat{\lambda}_{1}$ is simple (that is, $\operatorname{dim} E\left(\hat{\lambda}_{1}\right)=1$ ) and from (33) it is clear that the nontrivial elements of $E\left(\hat{\lambda}_{1}\right)$ do not change sign. All the other eigenvalues have nodal (sign changing) eigenfunctions. By $\hat{u}_{1}$ we denote the $L^{2}(\Omega)$-normalized (that 
is, $\left.\left\|\hat{u}_{1}\right\|_{2}=1\right)$ positive eigenfunction corresponding to $\hat{\lambda}_{1}$. If $\beta \in L^{s}(\Omega)$ with $s>N$, then $\hat{u_{1}} \in C_{+} \backslash\{0\}$ (see Wang [34]). In fact, using the Harnack inequality (see Pucci \& Serrin [29, p. 163]) we have $u_{1}(z)>0$ for all $z \in \Omega$. Finally, if $\beta^{+} \in L^{\infty}(\Omega)$, then by the Hopf theorem (see, for example, Pucci \& Serrin [29, p. 120]), we have that $\hat{u}_{1} \in \operatorname{int} C_{+}$. When $\beta \in L^{s}(\Omega)$ with $s>\frac{N}{2}$, all the eigenspaces have the "unique continuation property" (UCP for short). Namely, if $u \in E\left(\hat{\lambda}_{k}\right)$ and vanishes on a set of positive measure, then $u \equiv 0$.

A similar analysis can be conducted for a weighted version of problem (2). So, let $m \in L^{\infty}(\Omega)$ with $m \geqslant 0, m \neq 0$. We consider the following weighted linear eigenvalue problem:

$$
-\Delta u(z)+\beta(z) u(z)=\lambda m(z) u(z) \text { in } \Omega, \frac{\partial u}{\partial n}=0 \text { on } \partial \Omega .
$$

As was the case for problem (2), problem (6) above has a strictly increasing sequence $\left\{\hat{\lambda}_{k}(m)\right\}_{k \geqslant 1}$ of distinct eigenvalues such that $\hat{\lambda}_{k}(m) \rightarrow+\infty$ as $k \rightarrow \infty$. The first eigenvalue $\hat{\lambda}_{1}(m)>-\infty$ is simple and has eigenfunctions of constant sign. All the other eigenvalues have nodal eigenfunctions. The Rayleigh quotient is $\frac{\tau(u)}{\int_{\Omega} m|u|^{2} d z}$, and using it we have variational characterizations similar to those in (5). Again the eigenspaces $E\left(\hat{\lambda}_{k}(m)\right), k \geqslant 1$, have the UCP, and as a consequence of this property we obtain the following result.

Proposition 2. Assume that $m_{1}, m_{2} \in L^{\infty}(\Omega)_{+} \backslash\{0\}, m_{1}(z) \leqslant m_{2}(z)$ a.e. in $\Omega$, $m_{1} \neq m_{2}$. Then $\hat{\lambda}_{k}\left(m_{2}\right)<\hat{\lambda}_{k}\left(m_{1}\right)$ for all $k \geqslant 1$.

Let $\bar{H}_{k}=\bigoplus_{i=1}^{k} E\left(\hat{\lambda}_{i}\right)$ and $\hat{H}_{k}=\bar{H}_{k}^{\perp}=\overline{\bigoplus_{i \geqslant k+1} E\left(\hat{\lambda}_{i}\right)}$. As a consequence of the $\mathrm{UCP}$, we have the following simple but useful inequalities.

Proposition 3. (a) If $k \geqslant 0$ is an integer, $\vartheta \in L^{\infty}(\Omega)$ and $\vartheta(z) \leqslant \hat{\lambda}_{k+1}$ a.e. in $\Omega$, $\vartheta \neq \hat{\lambda}_{k+1}$, then there exists $\xi_{1}>0$ such that

$$
\tau(u)-\int_{\Omega} \vartheta u^{2} d z \geqslant \xi_{1}\|u\|^{2} \text { for all } u \in \hat{H}_{k} .
$$

(b) If $k \geqslant 1$ is an integer, $\eta \in L^{\infty}(\Omega)$ and $\eta(z) \geqslant \hat{\lambda}_{k}$ a.e. in $\Omega, \vartheta \neq \hat{\lambda}_{k}$, then there exists $\xi_{2}>0$ such that

$$
\tau(u)-\int_{\Omega} \eta u^{2} d z \leqslant-\xi_{2}\|u\|^{2} \text { for all } u \in \bar{H}_{k} .
$$

Next we recall some definitions and facts from Morse theory (critical groups) which we will need in the sequel.

So, let $X$ be a Banach space and $\varphi \in C^{1}(X), c \in \mathbb{R}$. We introduce the following sets:

$$
\begin{gathered}
\varphi^{c}=\{x \in X: \varphi(x) \leqslant c\}, K_{\varphi}=\left\{x \in X: \varphi^{\prime}(x)=0\right\} \\
\text { and } K_{\varphi}^{c}=\left\{x \in K_{\varphi}: \varphi(x)=c\right\} .
\end{gathered}
$$

Let $\left(Y_{1}, Y_{2}\right)$ be a topological pair such that $Y_{2} \subseteq Y_{1} \subseteq X$. For every integer $k \geqslant 0$, by $H_{k}\left(Y_{1}, Y_{2}\right)$ we denote the $\mathrm{k}^{t h}$-relative singular homology group for the pair $\left(Y_{1}, Y_{2}\right)$ with integer coefficients. Recall that for $k<0, H_{k}\left(Y_{1}, Y_{2}\right)=0$. The critical groups of $\varphi$ at an isolated critical point $u_{0} \in K_{\varphi}^{c}$ are defined by

$$
C_{k}\left(\varphi, u_{0}\right)=H_{k}\left(\varphi^{c} \cap U, \varphi^{c} \cap U \backslash\left\{u_{0}\right\}\right) \text { for every integer } k \geqslant 0 .
$$


Here $U$ is a neighborhood of $u_{0}$ such that $K_{\varphi} \cap \varphi^{c} \cap U=\left\{u_{0}\right\}$. The excision property of singular homology implies that this definition of critical groups is independent of the choice of the neighborhood $U$.

Suppose that $\varphi \in C^{1}(X)$ satisfies the $C$-condition and $\inf \varphi\left(K_{\varphi}\right)>-\infty$. Let $c<\inf \varphi\left(K_{\varphi}\right)$. Then the critical groups of $\varphi$ at infinity are defined by

$$
C_{k}(\varphi, \infty)=H_{k}\left(X, \varphi^{c}\right) \text { for all } k \geqslant 0 .
$$

The second deformation theorem (see, for example, Gasinski \& Papageorgiou [11, p. 628]) implies that this definition of critical groups at infinity is independent of the choice of the level $c<\inf \varphi\left(K_{\varphi}\right)$.

Suppose that $K_{\varphi}$ is finite. We introduce the following quantities:

$$
\begin{aligned}
& M(t, u)=\sum_{k \geqslant 0} \operatorname{rank} C_{k}(\varphi, u) t^{k} \text { for all } t \in \mathbb{R}, \text { all } u \in K_{\varphi}, \\
& P(t, \infty)=\sum_{k \geqslant 0} \operatorname{rank} C_{k}(\varphi, \infty) t^{k} \text { for all } t \in \mathbb{R} .
\end{aligned}
$$

The Morse relation says that

$$
\sum_{u \in K_{\varphi}} M(t, u)=P(t, \infty)+(1+t) Q(t)
$$

where $Q(t)=\sum_{k \geqslant 0} \beta_{k} t^{k}$ is a formal series in $t \in \mathbb{R}$ with nonnegative integer coefficients $\beta_{k}$.

Suppose that $X=Y \oplus V$ with $\operatorname{dim} Y<\infty$. We say that $\varphi \in C^{1}(X)$ admits a local linking at the origin, if there exist $\varrho>0$ such that

$$
\begin{aligned}
\varphi(y) & \leqslant 0 \text { for all } y \in Y \text { with }\|y\| \leqslant \varrho, \\
\text { and } \quad \varphi(v) & \geqslant 0 \text { for all } v \in V \text { with }\|v\| \leqslant \varrho .
\end{aligned}
$$

For functionals $\varphi$ with local linking at the origin, we have the following multiplicity result due to Brezis \& Nirenberg [5] (see also Liu \& Li [19]).

Proposition 4. Assume that $X=Y \oplus V$ with $\operatorname{dim} Y<\infty, \varphi \in C^{1}(X), \varphi(0)=0$, $\varphi$ is bounded below

$$
\inf _{X} \varphi<0
$$

and $\varphi$ satisfies the $C$-condition and admits local linking at the origin. Then $\varphi$ has at least two nontrivial critical points.

Throughout this work, our hypotheses on the potential function $\beta(\cdot)$ are the following:

$H_{0}: \beta \in L^{s}(\Omega)$ with $s>N$ and $\beta^{+} \in L^{\infty}(\Omega)$.

\section{THREE NONTRIVIAL SOLUTIONS}

In this section, by combining variational methods with the Lyapunov-Schmidt reduction technique and with Morse theory, we prove a "three solutions" theorem for problem (11), when resonance can occur at $\pm \infty$ with respect to any nonnegative nonprincipal eigenvalue of $\left(-\Delta-\beta, H^{1}(\Omega)\right)$. So, in what follows, $\hat{\lambda}_{m_{0}}$ denotes the first nonnegative eigenvalue of this operator. 
The hypotheses on the reaction term $f(z, x)$ are the following:

$H_{1}: \quad f: \Omega \times \mathbb{R} \rightarrow \mathbb{R}$ is a Carathéodory function such that $f(z, 0)=0$ for a.a. $z \in \Omega$ and

(i) there exist an integer $m \geqslant \max \left\{m_{0}, 2\right\}$ and a function $\eta \in L^{\infty}(\Omega)+$ such that

$$
\begin{aligned}
& \eta(z) \leqslant \hat{\lambda}_{m+1} \text { a.e. in } \Omega, \eta \neq \hat{\lambda}_{m+1}, \\
& (f(z, x)-f(z, y))(x-y) \leqslant \eta(z)(x-y)^{2} \text { for a.a. } z \in \Omega, \text { all } x, y \in \mathbb{R} ;
\end{aligned}
$$

(ii) $\hat{\lambda}_{m} \leqslant \liminf \lim _{x \rightarrow \pm \infty} \frac{f(z, x)}{x}$ uniformly for a.a. $z \in \Omega$;

(iii) if $F(z, x)=\int_{0}^{x} f(z, s) d s$, then we have

$$
\lim _{x \rightarrow \pm \infty}[f(z, x) x-2 F(z, x)]=-\infty \text { uniformly for a.a. } z \in \Omega
$$

(iv) there exists a function $\vartheta \in L^{\infty}(\Omega)$ such that

$$
\begin{aligned}
& \vartheta(z) \leqslant \hat{\lambda}_{1} \text { a.e. in } \Omega, \vartheta \neq \hat{\lambda}_{1}, \\
& \limsup _{x \rightarrow 0} \frac{2 F(z, x)}{x^{2}} \leqslant \vartheta(z) \text { uniformly for a.a. } z \in \Omega
\end{aligned}
$$

(v) for every $\varrho>0$, there exists $\xi_{\varrho}>0$ such that

$$
f(z, x) x+\xi_{\varrho} x^{2} \geqslant 0 \text { for a.a. } z \in \Omega \text {, all }|x| \leqslant \varrho .
$$

Remark 1 . Hypotheses $H_{1}(i),(i i)$ imply that asymptotically at $\pm \infty$, the quotient $\frac{f(z, x)}{x}$ is in the spectral interval $\left[\hat{\lambda}_{m}, \hat{\lambda}_{m+1}\right]$ with possible resonance with respect to $\hat{\lambda}_{m}$ (see $H_{1}(i i)$ ), while at the other end we have nonuniform nonresonance (see $\left.H_{1}(i)\right)$.

Example 1. The following function satisfies hypotheses $H_{1}$ above. For the sake of simplicity, we drop the $z$-dependence:

$$
f(x)= \begin{cases}\vartheta x+\xi|x|^{p-2} x & \text { if }|x| \leqslant 1 \\ \lambda x+\frac{c}{x} & \text { if } 1<|x|\end{cases}
$$

with $\vartheta<\hat{\lambda}_{1}, p>2, \xi=\lambda+c-\vartheta, \lambda \in\left[\hat{\lambda}_{m}, \hat{\lambda}_{m+1}\right)$ for some integer $m \geqslant \max \left\{m_{0}, 2\right\}$, $c>0,2 c<\lambda$.

Let $\xi_{0}>0$ be as in (44) and consider the following truncation-perturbation of the reaction $f(z, \cdot)$ :

$$
\hat{f}_{ \pm}(z, x)=f\left(z, \pm x^{ \pm}\right)+\xi_{0}\left( \pm x^{ \pm}\right) .
$$

Both are Carathéodory functions. We set $\hat{F}_{ \pm}(z, x)=\int_{0}^{x} \hat{f}_{ \pm}(z, s) d s$ and introduce the $C^{1}$ - functionals $\varphi, \hat{\varphi}_{ \pm}: H^{1}(\Omega) \rightarrow \mathbb{R}$ defined by

$$
\begin{aligned}
& \varphi(u)=\frac{1}{2} \tau(u)-\int_{\Omega} F(z, u(z)) d z, \\
& \hat{\varphi}_{ \pm}(u)=\frac{1}{2} \tau(u)+\frac{\xi_{0}}{2}\|u\|_{2}^{2}-\int_{\Omega} \hat{F}_{ \pm}(z, u(z)) d z \text { for all } u \in H^{1}(\Omega) .
\end{aligned}
$$

Proposition 5. If hypotheses $H_{0}$ and $H_{1}$ hold, then the functionals $\hat{\varphi}_{ \pm}$satisfy the C-condition. 
Proof. Let $\left\{u_{n}\right\}_{n \geqslant 1} \subseteq H^{1}(\Omega)$ be a sequence such that

$$
\left|\hat{\varphi}_{+}\left(u_{n}\right)\right| \leqslant M_{1} \text { for some } M_{1}>0, \text { all } n \geqslant 1,
$$$$
\text { and }\left(1+\left\|u_{n}\right\|\right) \hat{\varphi}_{+}^{\prime}\left(u_{n}\right) \rightarrow 0 \text { in } H^{1}(\Omega)^{*} \text { as } n \rightarrow \infty .
$$

From (9) we have

$$
\begin{array}{r}
\left|\left\langle A\left(u_{n}\right), h\right\rangle+\int_{\Omega}\left(\beta(z)+\xi_{0}\right) u_{n} h d z-\int_{\Omega} \hat{f}_{+}\left(z, u_{n}\right) h d z\right| \leqslant \frac{\varepsilon_{n}\|h\|}{1+\left\|u_{n}\right\|} \\
\text { for all } h \in H^{1}(\Omega) \text { with } \varepsilon_{n} \rightarrow 0^{+} .
\end{array}
$$

Here $A \in \mathcal{L}\left(H^{1}(\Omega), H^{1}(\Omega)^{*}\right)$ is defined by

$$
\langle A(u), y\rangle=\int_{\Omega}(D u, D y)_{\mathbb{R}^{N}} d z \text { for all } u, y \in H^{1}(\Omega) .
$$

In (10) we choose $h=-u_{n}^{-} \in H^{1}(\Omega)$ and obtain

$$
\begin{aligned}
& \tau\left(u_{n}^{-}\right)+\xi_{0}\left\|u_{n}^{-}\right\|_{2}^{2} \leqslant \varepsilon_{n} \text { for all } n \geqslant 1, \\
\Rightarrow & \left.c_{1}\left\|u_{n}^{-}\right\|^{2} \leqslant \varepsilon_{n} \text { for all } n \geqslant 1 \text { (see (4) }\right), \\
\Rightarrow & u_{n}^{-} \rightarrow 0 \text { in } H^{1}(\Omega) .
\end{aligned}
$$

If we use (11) in (10), then

$$
\begin{array}{r}
\left|\left\langle A\left(u_{n}^{+}\right), h\right\rangle+\int_{\Omega} \beta(z) u_{n}^{+} h d z-\int_{\Omega} f\left(z, u_{n}^{+}\right) h d z\right| \leqslant \varepsilon_{n}^{\prime}\|h\| \\
\text { for all } h \in H^{1}(\Omega) \text { with } \varepsilon_{n}^{\prime} \downarrow 0 .
\end{array}
$$

Suppose that $\left\|u_{n}^{+}\right\| \rightarrow \infty$. We set $y_{n}=\frac{u_{n}^{+}}{\left\|u_{n}^{+}\right\|}, n \geqslant 1$. Then $\left\|y_{n}\right\|=1$ for all $n \geqslant 1$, and so we may assume that

$$
y_{n} \stackrel{w}{\longrightarrow} y \text { in } H^{1}(\Omega) \text { and } y_{n} \rightarrow y \text { in } L^{2 s^{\prime}}(\Omega)\left(\frac{1}{s}+\frac{1}{s^{\prime}}=1\right) .
$$

Multiplying (12) with $\frac{1}{\left\|u_{n}^{+}\right\|}$, we obtain

$$
\left|\left\langle A\left(y_{n}\right), h\right\rangle+\int_{\Omega} \beta(z) y_{n} h d z-\int_{\Omega} \frac{N_{f}\left(u_{n}^{+}\right)}{\left\|u_{n}^{+}\right\|} h d z\right| \leqslant \varepsilon_{n}^{\prime \prime}\|h\| \text { with } \varepsilon_{n}^{\prime \prime} \rightarrow 0 .
$$

From hypothesis $H_{1}(i)$, we have

$$
f(z, x) \leqslant \eta(z) x \text { for a.a. } z \in \Omega, \text { all } x \geqslant 0 .
$$

On the other hand hypotheses $H_{1}(i i)$, (iv) imply that we can find $c_{2}>0$ such that

$$
-c_{2} x \leqslant f(z, x) \text { for a.a. } z \in \Omega \text {, all } x \geqslant 0 .
$$

From (15) and (16) it follows that

$$
\begin{aligned}
& -c_{2} y_{n}(z) \leqslant \frac{f\left(z, u_{n}^{+}(z)\right)}{\left\|u_{n}^{+}\right\|} \leqslant \eta(z) y_{n}(z) \text { a.e. in } \Omega \\
\Rightarrow & \left\{\frac{N_{f}\left(u_{n}^{+}\right)}{\left\|u_{n}^{+}\right\|}\right\}_{n \geqslant 1} \subseteq L^{2}(\Omega) \text { is bounded. }
\end{aligned}
$$


Therefore, by passing to a subsequence if necessary and using hypotheses $H_{1}(i i)$ and (17), we have

$$
\frac{N_{f}\left(u_{n}^{+}\right)}{\left\|u_{n}^{+}\right\|} \stackrel{w}{\longrightarrow} \hat{\xi} y \text { in } L^{2}(\Omega),
$$

with $\hat{\lambda}_{m} \leqslant \hat{\xi}(z) \leqslant \eta(z)$ a.e. in $\Omega$ (see Aizicovici, Papageorgiou \& Staicu 1, proof of Proposition 30). In (14) we choose $h=y_{n}-y \in H^{1}(\Omega)$ and pass to the limit as $n \rightarrow \infty$. Using (13) and (18), we obtain

$$
\begin{aligned}
& \lim _{n \rightarrow \infty}\left\langle A\left(y_{n}\right), y_{n}-y\right\rangle=0, \\
\Rightarrow \quad & \left\|D y_{n}\right\|_{2} \rightarrow\|D y\|_{2}, \\
\Rightarrow \quad & y_{n} \rightarrow y \text { in } H^{1}(\Omega), \text { hence }\|y\|=1, y \geqslant 0 \\
& \text { (see (13) and use the Kadec-Klee property). }
\end{aligned}
$$

In (14) we pass to the limit as $n \rightarrow \infty$ and use (18) and (19). Then

$$
\begin{aligned}
& \langle A(y), h\rangle+\int_{\Omega} \beta y h d z=\int_{\Omega} \hat{\xi} y h d z \text { for all } h \in H^{1}(\Omega), \\
\Rightarrow & A(y)+\beta y=\hat{\xi} y, \\
\Rightarrow & -\Delta y(z)+\beta(z) y(z)=\hat{\xi}(z) y(z) \text { a.e. in } \Omega, \frac{\partial y}{\partial n}=0 \text { on } \partial \Omega .
\end{aligned}
$$

From the properties of $\hat{\xi}$ and Proposition 2, we have

$$
\hat{\lambda}_{1}(\hat{\xi})<\hat{\lambda}_{1}\left(\hat{\lambda}_{1}\right)=1 .
$$

Since $y \neq 0$ (see (19)), from (20) and (21) it follows that $y$ must be nodal, a contradiction to (19). This proves that the sequence $\left\{u_{n}\right\}_{n \geqslant 1} \subseteq H^{1}(\Omega)$ is bounded, and so we may assume that

$$
u_{n} \stackrel{w}{\longrightarrow} u \text { in } H^{1}(\Omega) \text { and } u_{n} \rightarrow u \text { in } L^{2 s^{\prime}}(\Omega) .
$$

In (10), we choose $h=u_{n}-u \in H^{1}(\Omega)$, pass to the limit as $n \rightarrow \infty$ and use (22). Then we obtain

$$
\begin{aligned}
& \lim _{n \rightarrow \infty}\left\langle A\left(u_{n}\right), u_{n}-u\right\rangle=0, \\
\Rightarrow & u_{n} \rightarrow u \text { in } H^{1}(\Omega) \text { (by the Kadec-Klee property), } \\
\Rightarrow \quad & \hat{\varphi}_{+} \text {satisfies the } C \text {-condition. }
\end{aligned}
$$

Similarly for the functional $\hat{\varphi}_{-}$.

Proposition 6. If hypotheses $H_{0}$ and $H_{1}$ hold, then the functional $\varphi$ satisfies the C-condition.

Proof. Let $\left\{u_{n}\right\}_{n \geqslant 1} \subseteq H^{1}(\Omega)$ be a sequence such that

$$
\begin{aligned}
& \qquad\left|\varphi\left(u_{n}\right)\right| \leqslant M_{2} \text { for some } M_{2}>0, \text { all } n \geqslant 1, \\
& \text { and }\left(1+\left\|u_{n}\right\|\right) \varphi^{\prime}\left(u_{n}\right) \rightarrow 0 \text { in } H^{1}(\Omega)^{*} \text { as } n \rightarrow \infty .
\end{aligned}
$$

From (24) we have

$$
\begin{array}{r}
\left|\left\langle A\left(u_{n}\right), h\right\rangle+\int_{\Omega} \beta(z) u_{n} h d z-\int_{\Omega} f\left(z, u_{n}\right) h d z\right| \leqslant \frac{\varepsilon_{n}\|h\|}{1+\left\|u_{n}\right\|} \\
\text { for all } h \in H^{1}(\Omega) \text { with } \varepsilon_{n} \rightarrow 0^{+} .
\end{array}
$$


Claim 1. $\left\{u_{n}\right\}_{n \geqslant 1} \subseteq H^{1}(\Omega)$ is bounded.

Arguing by contradiction, suppose that $\left\|u_{n}\right\| \rightarrow \infty$. We set $y_{n}=\frac{u_{n}}{\left\|u_{n}\right\|}, n \geqslant 1$. Then $\left\|y_{n}\right\|=1$ for all $n \geqslant 1$, and so we may assume that

$$
y_{n} \stackrel{w}{\longrightarrow} y \text { in } H^{1}(\Omega) \text { and } y_{n} \rightarrow y \text { in } L^{2 s^{\prime}}(\Omega) .
$$

From (25), we have

$$
\left|\left\langle A\left(y_{n}\right), h\right\rangle+\int_{\Omega} \beta(z) y_{n} h d z-\int_{\Omega} \frac{N_{f}\left(u_{n}\right)}{\left\|u_{n}\right\|} h d z\right| \leqslant \varepsilon_{n} \text { for all } n \geqslant 1 .
$$

As in the proof of Proposition 5 , using hypotheses $H_{1}(i),(i i),(i v)$, we see that $\left\{\frac{N_{f}\left(u_{n}\right)}{\left\|u_{n}\right\|}\right\}_{n \geqslant 1} \subseteq L^{2}(\Omega)$ is bounded. Hence, by passing to a subsequence if necessary and using hypotheses $H_{1}(i),(i i)$, we can say that

$$
\frac{N_{f}\left(u_{n}\right)}{\left\|u_{n}\right\|} \stackrel{w}{\longrightarrow} \hat{\xi} y \text { in } L^{2}(\Omega) \text { with } \hat{\lambda}_{m} \leqslant \hat{\xi}(z) \leqslant \eta(z) \text { a.e. in } \Omega \text {. }
$$

Choosing $h=y_{n}-y \in H^{1}(\Omega)$ in (27), passing to the limit as $n \rightarrow \infty$ and using (26) and (28), we obtain

$$
\lim _{n \rightarrow \infty}\left\langle A\left(y_{n}\right), y_{n}-y\right\rangle=0
$$

(29) $\Rightarrow y_{n} \rightarrow y$ in $H^{1}(\Omega)$ (by the Kadec-Klee property), hence $\|y\|=1$.

So, if in (27) we pass to the limit as $n \rightarrow \infty$ and use (28) and (29), then

$$
\begin{aligned}
& \langle A(y), h\rangle+\int_{\Omega} \beta(z) y h d z=\int_{\Omega} \hat{\xi} y h d z \text { for all } h \in H^{1}(\Omega), \\
\Rightarrow \quad & A(y)+\beta y=\hat{\xi} y, \\
\Rightarrow & -\Delta y(z)+\beta(z) y(z)=\hat{\xi}(z) y(z) \text { a.e. in } \Omega, \frac{\partial y}{\partial n}=0 \text { on } \partial \Omega .
\end{aligned}
$$

From (29) we know that $y \neq 0$. Recall that $\hat{\lambda}_{m} \leqslant \hat{\xi}(z) \leqslant \eta(z)$ a.e. in $\Omega$. If $\hat{\xi} \neq \hat{\lambda}_{m}$, then from (30) it follows that $y=0$, a contradiction. If $\hat{\xi}=\hat{\lambda}_{m}$, then from (30) we see that $y \in E\left(\hat{\lambda}_{m}\right) \backslash\{0\}$, and so by the UCP we have that $y(z) \neq 0$ for a.a. $z \in \Omega$ and so $\left|u_{n}(z)\right| \rightarrow+\infty$ for a.a. $z \in \Omega$. Then by virtue of hypothesis $H_{1}(i i i)$, we have

$$
\begin{gathered}
f\left(z, u_{n}(z)\right) u_{n}(z)-2 F\left(z, u_{n}(z)\right) \rightarrow-\infty \text { for a.a. } z \in \Omega, \\
\left.(31) \Rightarrow \int_{\Omega}\left[f\left(z, u_{n}\right) u_{n}-2 F\left(z, u_{n}\right)\right] d z \rightarrow-\infty \text { (by Fatou's lemma; see } H_{1}(i i i)\right) .
\end{gathered}
$$

From (23) we have

$$
\tau\left(u_{n}\right)-\int_{\Omega} 2 F\left(z, u_{n}\right) d z \geqslant-2 M_{2} \text { for all } n \geqslant 1 .
$$

In (25) we choose $h=u_{n} \in H^{1}(\Omega)$ and obtain

$$
-\tau\left(u_{n}\right)+\int_{\Omega} f\left(z, u_{n}\right) u_{n} d z \geqslant-\varepsilon_{n} \text { for all } n \geqslant 1 .
$$

Adding (32) and (33), we obtain

$$
\int_{\Omega}\left[f\left(z, u_{n}\right) u_{n}-2 F\left(z, u_{n}\right)\right] d z \geqslant-M_{3} \text { for some } M_{3}>0, \text { all } n \geqslant 1 \text {. }
$$

Comparing (31) and (34), we reach a contradiction. This proves the claim. 
By virtue of the claim, we may assume that

$$
u_{n} \stackrel{w}{\longrightarrow} u \text { in } H^{1}(\Omega) \text { and } u_{n} \rightarrow u \text { in } L^{2 s^{\prime}}(\Omega) .
$$

From (25) with $h=u_{n}-u \in H^{1}(\Omega)$ and using (35) we have

$$
\lim _{n \rightarrow \infty}\left\langle A\left(u_{n}\right), u_{n}-u\right\rangle=0,
$$

$\Rightarrow \quad u_{n} \rightarrow u$ in $H^{1}(\Omega)$ (by the Kadec-Klee property),

$\Rightarrow \varphi$ satisfies the $C$-condition.

Proposition 7. If hypotheses $H_{0}$ and $H_{1}$ hold, then $u=0$ is a minimizer for the functionals $\hat{\varphi}_{ \pm}$and $\varphi$.

Proof. We do the proof for $\hat{\varphi}_{+}$, with the proofs for $\hat{\varphi}_{-}$and $\varphi$ being similar.

By virtue of hypothesis $H_{1}(i v)$, given $\varepsilon>0$ we can find $\delta=\delta(\varepsilon)>0$ such that

$$
F(z, x) \leqslant \frac{1}{2}(\vartheta(z)+\varepsilon) x^{2} \text { for a.a. } z \in \Omega, \text { all }|x| \leqslant \delta .
$$

Then for $u \in C^{1}(\bar{\Omega})$ with $\|u\|_{C^{1}(\bar{\Omega})} \leqslant \delta$, we have

$$
\begin{aligned}
\hat{\varphi}_{+}(u) & =\frac{1}{2} \tau(u)+\frac{\xi_{0}}{2}\|u\|_{2}^{2}-\int_{\Omega} \hat{F}_{+}(z, u) d z \\
& \geqslant \frac{1}{2}\left[\tau(u)-\int_{\Omega} \vartheta u^{2} d z-\varepsilon\|u\|^{2}\right] \quad(\text { see }(\underline{36})) \\
& \geqslant \frac{\xi_{1}-\varepsilon}{2}\|u\|^{2}(\text { see Proposition }[3(a)) .
\end{aligned}
$$

Choosing $\varepsilon \in\left(0, \xi_{1}\right)$, from (37) it follows that $u=0$ is a local $C^{1}(\bar{\Omega})$-minimizer of $\hat{\varphi}_{+}$. From Motreanu \& Papageorgiou [24, it follows that $u=0$ is a local $H^{1}(\Omega)$ minimizer of $\hat{\varphi}_{+}$. Similarly for the functionals $\hat{\varphi}_{-}, \varphi$.

Proposition 8. If hypotheses $H_{0}$ and $H_{1}$ hold, then $\hat{\varphi}_{ \pm}\left(t \hat{u}_{1}\right) \rightarrow-\infty$ as $t \rightarrow \pm \infty$.

Proof. By virtue of hypotheses $H_{1}(i),(i i),(v)$, given $\varepsilon>0$ we can find $c_{2}=c_{2}(\varepsilon)>$ 0 such that

$$
F(z, x) \leqslant \frac{1}{2}\left[\hat{\lambda}_{m}-\varepsilon\right] x^{2}-c_{2} \text { for a.a. } z \in \Omega, \text { all } x \in \mathbb{R} .
$$

Then for $t>0$, we have

$$
\begin{aligned}
\hat{\varphi}_{+}\left(t \hat{u}_{1}\right) & =\frac{t^{2}}{2} \hat{\lambda}_{1}-\int_{\Omega} F\left(z, t \hat{u}_{1}\right) d z \\
& \leqslant \frac{t^{2}}{2}\left[\hat{\lambda}_{1}+\varepsilon-\hat{\lambda}_{m}\right]+c_{2}|\Omega|_{N}(\text { see (38) }) .
\end{aligned}
$$

We choose $\varepsilon \in\left(0, \hat{\lambda}_{m}-\hat{\lambda}_{1}\right)$ (recall that $\left.m \geqslant 2\right)$. Then from (39) it follows that

$$
\hat{\varphi}_{+}\left(t \hat{u}_{1}\right) \rightarrow-\infty \text { as } t \rightarrow+\infty \text {. }
$$

Similarly, we show that

$$
\hat{\varphi}_{-}\left(t \hat{u}_{1}\right) \rightarrow-\infty \text { as } t \rightarrow+\infty \text {. }
$$


Now we are ready to produce two nontrivial solutions of constant sign for problem (11).

Proposition 9. If hypotheses $H_{0}$ and $H_{1}$ hold, then problem (11) admits at least two nontrivial constant sign solutions

$$
u_{0} \in \operatorname{int} C_{+} \text {and } v_{0} \in-i n t C_{+} .
$$

Proof. According to Proposition [7 $u=0$ is a local minimizer of the functional $\hat{\varphi}_{+}$. So, we can find $\varrho \in(0,1)$ small such that

$$
\hat{\varphi}_{+}(0)=0<\inf \left[\hat{\varphi}_{+}(u):\|u\|=\varrho\right]=\hat{\eta}_{\varrho}^{+}
$$

(see Aizicovici, Papageorgiou \& Staicu 1] (proof of Proposition 29)). Then relation (40), together with Propositions 5and 8, permit the use of Theorem[1 (the mountain pass theorem). So, we can find $u_{0} \in H^{1}(\Omega)$ such that

$$
\hat{\eta}_{\varrho}^{+} \leqslant \hat{\varphi}_{+}\left(u_{0}\right) \text { and } \hat{\varphi}_{+}^{\prime}\left(u_{0}\right)=0 .
$$

From (40) and (41) it follows that

$$
A\left(u_{0}\right)+\left(\beta+\xi_{0}\right) u_{0}=N_{\hat{f}_{+}}\left(u_{0}\right), u_{0} \neq 0 .
$$

On (42) we act with $-u_{0}^{-} \in H^{1}(\Omega)$, and using (4), we obtain $u_{0} \geqslant 0, u_{0} \neq 0$. So, we have

$$
\begin{aligned}
& A\left(u_{0}\right)+\beta u_{0}=N_{f}\left(u_{0}\right), \\
\Rightarrow \quad & -\Delta u_{0}(z)+\beta(z) u_{0}(z)=f\left(z, u_{0}(z)\right) \text { a.e. in } \Omega, \frac{\partial u_{0}}{\partial n}=0 \text { on } \partial \Omega .
\end{aligned}
$$

Thus, $u_{0}$ is a nontrivial positive solution of (1). We set

$$
k(z)= \begin{cases}\frac{f\left(z, u_{0}(z)\right)}{u_{0}(z)} & \text { if } u_{0}(z) \neq 0 \\ 0 & \text { otherwise }\end{cases}
$$

Then $k \in L^{\infty}(\Omega)$. We have

$$
-\Delta u_{0}(z)=(k-\beta)(z) u_{0}(z) \text { a.e. in } \Omega, \frac{\partial u_{0}}{\partial n}=0 \text { on } \partial \Omega \text {. }
$$

Note that $k-\beta \in L^{s}(\Omega)$ (see $H_{0}$ and recall that $k \in L^{\infty}(\Omega)$ ). Then from Lemma 5.1 of Wang [34, we have that $u_{0} \in L^{\infty}(\Omega)$. From (43) it follows that $\Delta u_{0} \in L^{s}(\Omega)$, and so Lemma 5.2 of Wang 34 implies that $u_{0} \in W^{2, s}(\Omega)$. Since $s>N$ (see hypothesis $H_{0}$ ), by virtue of the Sobolev embedding theorem, we have

$$
W^{2, s}(\Omega) \hookrightarrow C^{1+\alpha}(\bar{\Omega}) \text { with } \alpha=1-\frac{N}{s}>0 .
$$

Therefore, we infer that $u_{0} \in C_{+} \backslash\{0\}$. From (43) we have

$$
\begin{aligned}
& \Delta u_{0}(z)=(\beta-k)(z) u_{0}(z) \leqslant\left(\left\|\beta^{+}\right\|_{\infty}+\|k\|_{\infty}\right) u_{0}(z) \text { a.e. in } \Omega, \\
\Rightarrow \quad & u_{0} \in \operatorname{int} C_{+}(\text {see Vazquez [33] }) .
\end{aligned}
$$

In a similar fashion, working this time with $\hat{\varphi}_{-}$, we produce a second nontrivial constant sign solution $v_{0} \in-\operatorname{int} C_{+}$.

To produce a third nontrivial solution, we will employ the so-called LyapunovSchmidt reduction technique, as this was formulated for elliptic equations by Amann [2], Castro \& Lazer [7, and Thews [32]. 
To this end, we introduce the following subspaces of $H^{1}(\Omega)$ :

$$
Y=\bigoplus_{i=1}^{m} E\left(\hat{\lambda}_{i}\right) \text { and } \hat{H}=Y^{\perp}=\bigoplus_{i \geqslant m+1} E\left(\hat{\lambda}_{i}\right) .
$$

We have the following orthogonal direct sum decomposition:

$$
H^{1}(\Omega)=Y \oplus \hat{H} .
$$

The reduction method will be based on this decomposition.

Proposition 10. If hypotheses $H_{0}$ and $H_{1}$ hold, then there exists a continuous map $\gamma_{0}: Y \rightarrow \hat{H}$ such that

$$
\varphi\left(y+\gamma_{0}(y)\right)=\inf [\varphi(y+\hat{u}): \hat{u} \in \hat{H}] \text { for all } y \in Y .
$$

Proof. We fix $y \in Y$ and consider the $C^{1}$-functional $\varphi_{y}: H^{1}(\Omega) \rightarrow \mathbb{R}$ defined by

$$
\varphi_{y}(u)=\varphi(y+u) \text { for all } u \in H^{1}(\Omega) .
$$

Let $i: \hat{H} \rightarrow H^{1}(\Omega)$ be the inclusion map and set $\hat{\varphi}_{y}=\varphi_{y} \circ i: \hat{H} \rightarrow \mathbb{R}$. The chain rule implies that

$$
\hat{\varphi}_{y}^{\prime}(\hat{u})=p_{\hat{H}^{*}} \varphi_{y}^{\prime}(\hat{u}) \text { for all } \hat{u} \in \hat{H}
$$

where $p_{\hat{H}^{*}}$ is the orthogonal projection of $H^{1}(\Omega)$ onto $\hat{H}^{*}$. Let $\langle\cdot, \cdot\rangle_{\hat{H}}$ denote the duality brackets for the pair $\left(\hat{H}^{*}, \hat{H}\right)$. For $\hat{u}_{1}, \hat{u}_{2} \in \hat{H}$ we have

$$
\begin{aligned}
& \left\langle\hat{\varphi}_{y}^{\prime}\left(\hat{u}_{1}\right)-\hat{\varphi}_{y}^{\prime}\left(\hat{u}_{2}\right), \hat{u}_{1}-\hat{u}_{2}\right\rangle_{\hat{H}} \\
= & \left\langle\varphi_{y}^{\prime}\left(\hat{u}_{1}\right)-\varphi_{y}^{\prime}\left(\hat{u}_{2}\right), \hat{u}_{1}-\hat{u}_{2}\right\rangle \\
= & \left\langle A\left(\hat{u}_{1}\right)-A\left(\hat{u}_{2}\right), \hat{u}_{1}-\hat{u}_{2}\right\rangle+\int_{\Omega} \beta(z)\left(\hat{u}_{1}-\hat{u}_{2}\right)^{2} d z \\
- & \int_{\Omega}\left[f\left(z, y+\hat{u}_{1}\right)-f\left(z, y+\hat{u}_{2}\right)\right]\left(\hat{u}_{1}-\hat{u}_{2}\right) d z \\
\geqslant & \tau\left(\hat{u}_{1}-\hat{u}_{2}\right)-\int_{\Omega} \eta\left(\hat{u}_{1}-\hat{u}_{2}\right)^{2} d z\left(\text { see hypothesis } H_{1}(i)\right)
\end{aligned}
$$

$$
\begin{gathered}
\left.\geqslant \xi_{1}\left\|\hat{u}_{1}-\hat{u}_{2}\right\|^{2} \text { (see Proposition } 3(a) \text { and recall that } \hat{u}_{1}, \hat{u}_{2} \in \hat{H}\right) \\
\Rightarrow \hat{\varphi}_{y}^{\prime} \text { is strongly monotone, hence } \hat{\varphi}_{y} \text { is strictly convex. }
\end{gathered}
$$

Also, note that

$$
\begin{aligned}
\left\langle\hat{\varphi}_{y}^{\prime}(\hat{u}), \hat{u}\right\rangle_{\hat{H}} & =\left\langle\hat{\varphi}_{y}^{\prime}(\hat{u})-\hat{\varphi}_{y}^{\prime}(0), \hat{u}\right\rangle_{\hat{H}}+\left\langle\hat{\varphi}_{y}(0), \hat{u}\right\rangle_{\hat{H}} \\
& \geqslant \xi_{1}\|\hat{u}\|^{2}-c_{3}\|\hat{u}\| \text { for some } c_{3}>0(\text { see (44) }), \\
\Rightarrow \hat{\varphi}_{y}^{\prime} \text { is coercive. } &
\end{aligned}
$$

Since $\hat{\varphi}_{y}^{\prime}$ is strongly monotone (hence maximal monotone too, being continuous) and coercive, we have that $\hat{\varphi}_{y}^{\prime}$ is surjective (see, for example, Papageorgiou \& Kyritsi [26, p. 172]). So, we can find $\hat{u}_{0} \in \hat{H}$ such that

$$
\hat{\varphi}_{y}^{\prime}\left(\hat{u}_{0}\right)=0 \text {. }
$$


In fact, relation (45) implies that $\hat{u}_{0} \in \hat{H}$ is unique and is the unique minimizer on $\hat{H}$ of the strictly convex functional $\hat{\varphi}_{y}=\left.\varphi_{y}\right|_{\hat{H}}$. So, we can define the map $\gamma_{0}: Y \rightarrow \hat{H}$ by setting $\gamma_{0}(y)=\hat{u}_{0}$. We have

$$
p_{\hat{H}^{*}} \varphi^{\prime}\left(y+\gamma_{0}(y)\right)=0 \text { and } \varphi\left(y+\gamma_{0}(y)\right)=\inf [\varphi(y+\hat{u}): \hat{u} \in \hat{H}] .
$$

Next we examine the continuity properties of the map $y \mapsto \gamma_{0}(y)$. To this end, suppose that $y_{n} \rightarrow y$ in $Y$. For every $n \geqslant 1$, we have

$$
\begin{aligned}
0 & =\left\langle\hat{\varphi}_{y_{n}}^{\prime}\left(\gamma_{0}\left(y_{n}\right)\right), \gamma_{0}\left(y_{n}\right)\right\rangle_{\hat{H}}(\text { see (47)) } \\
& \geqslant \xi_{1}\left\|\gamma_{0}\left(y_{n}\right)\right\|^{2}-c_{3}\left\|\gamma_{0}\left(y_{n}\right)\right\|(\text { see (46) }), \\
& \Rightarrow\left\{\gamma_{0}\left(y_{n}\right)\right\}_{n \geqslant 1} \subseteq \hat{H} \text { is bounded. }
\end{aligned}
$$

So, at least for a subsequence we have

$$
\gamma_{0}\left(y_{n}\right) \stackrel{w}{\longrightarrow} h \text { in } H^{1}(\Omega) \text { and } h \in \hat{H} .
$$

Using the Sobolev embedding theorem, we deduce that $\varphi$ is sequentially weakly lower semicontinuous. Hence

$$
\varphi(y+h) \leqslant \liminf _{n \rightarrow \infty} \varphi\left(y_{n}+\gamma_{0}\left(y_{n}\right)\right) .
$$

From (48), we have

$$
\begin{aligned}
& \varphi\left(y_{n}+\gamma_{0}\left(y_{n}\right)\right) \leqslant \varphi\left(y_{n}+\hat{u}\right) \text { for all } \hat{u} \in \hat{H}, \text { all } n \geqslant 1, \\
\Rightarrow & \varphi(y+h) \leqslant \varphi(y+\hat{u}) \text { for all } \hat{u} \in \hat{H}, \\
\Rightarrow & h=\gamma_{0}(y) \\
\Rightarrow & \gamma_{0}\left(y_{n}\right) \stackrel{w}{\longrightarrow} \gamma_{0}(y) \text { in } H^{1}(\Omega) .
\end{aligned}
$$

Also, we have

$$
\begin{aligned}
& p_{\hat{H}^{*}} \varphi^{\prime}\left(y_{n}+\gamma_{0}\left(y_{n}\right)\right)=0 \text { for all } n \geqslant 1(\text { see (47)) }, \\
\Rightarrow \quad & p_{\hat{H}^{*}}\left[A\left(y_{n}+\gamma_{0}\left(y_{n}\right)\right)+\beta\left(y_{n}+\gamma_{0}\left(y_{n}\right)\right)\right]=p_{\hat{H}^{*}} N_{f}\left(y_{n}+\gamma_{0}\left(y_{n}\right)\right) \text { for all } n \geqslant 1 .
\end{aligned}
$$

On this equation, we act with $\gamma_{0}\left(y_{n}\right)-\gamma_{0}(y)$ and obtain

$$
\begin{aligned}
& \left\langle A\left(y_{n}+\gamma_{0}\left(y_{n}\right)\right), \gamma_{0}\left(y_{n}\right)-\gamma_{0}(y)\right\rangle+\int_{\Omega} \beta(z)\left(y_{n}+\gamma_{0}\left(y_{n}\right)\right)\left(\gamma_{0}\left(y_{n}\right)-\gamma_{0}(y)\right) d z \\
= & \int_{\Omega} f\left(z, y_{n}+\gamma_{0}\left(y_{n}\right)\right)\left(\gamma_{0}\left(y_{n}\right)-\gamma_{0}(y)\right) d z \text { for all } n \geqslant 1 .
\end{aligned}
$$

Passing to the limit as $n \rightarrow \infty$ and using (50), we obtain

$$
\begin{aligned}
& \lim _{n \rightarrow \infty}\left\langle A\left(y_{n}+\gamma_{0}\left(y_{n}\right)\right), \gamma_{0}\left(y_{n}\right)-\gamma_{0}(y)\right\rangle=0, \\
\Rightarrow & \left\|D\left(y_{n}+\gamma_{0}\left(y_{n}\right)\right)\right\|_{2} \rightarrow\left\|D\left(y+\gamma_{0}(y)\right)\right\|_{2}, \\
\Rightarrow & y_{n}+\gamma_{0}\left(y_{n}\right) \rightarrow y+\gamma_{0}(y) \text { in } H^{1}(\Omega) \text { (by the Kadec-Klee property), } \\
\Rightarrow & \gamma_{0}\left(y_{n}\right) \rightarrow \gamma_{0}(y) \text { in } H^{1}(\Omega), \\
\Rightarrow & \gamma_{0} \text { is continuous (by Urysohn's convergence criterion). }
\end{aligned}
$$

Let $\psi(y)=\varphi\left(y+\gamma_{0}(y)\right)$ for all $y \in Y$. The next proposition is not immediately clear, since $\gamma_{0}$ is only continuous.

Proposition 11. If hypotheses $H_{0}$ and $H_{1}$ hold, then $\psi \in C^{1}(Y)$. 
Proof. Let $y, v \in Y$ and $t>0$ (the analysis is similar if $t<0$ ). We have

$$
\begin{aligned}
& \frac{\psi(y+t v)-\psi(y)}{t} \leqslant \frac{\varphi\left(y+t v+\gamma_{0}(y)\right)-\varphi\left(y+\gamma_{0}(y)\right)}{t}, \\
\Rightarrow \quad & \limsup _{t \rightarrow 0} \frac{\psi(y+t v)-\psi(y)}{t} \leqslant\left\langle\varphi^{\prime}\left(y+\gamma_{0}(y)\right), v\right\rangle .
\end{aligned}
$$

Also, we have

$$
\begin{aligned}
& \frac{\psi(y+t v)-\psi(y)}{t} \geqslant \frac{\varphi\left(y+t v+\gamma_{0}(y+t v)\right)-\varphi\left(y+\gamma_{0}(y+t v)\right)}{t}, \\
\Rightarrow & \liminf _{t \rightarrow 0} \frac{\psi(y+t v)-\psi(y)}{t} \geqslant\left\langle\varphi^{\prime}\left(y+\gamma_{0}(y)\right), v\right\rangle
\end{aligned}
$$

(since $\varphi \in C^{1}\left(H^{1}(\Omega)\right)$ and $\gamma_{0}$ is continuous (see Proposition [10) ).

From (51) and (52) it follows that

$$
\psi \in C^{1}(Y) \text { and }\left\langle\psi^{\prime}(y), v\right\rangle_{Y}=\left\langle\varphi^{\prime}\left(y+\gamma_{0}(y)\right), v\right\rangle \text { for all } y, v \in Y .
$$

Proposition 12. If hypotheses $H_{0}$ and $H_{1}$ hold, then $\psi$ is anticoercive (that is, if $\|y\| \rightarrow \infty$, then $\psi(y) \rightarrow-\infty)$.

Proof. We argue indirectly. So, suppose that we can find $\left\{y_{n}\right\}_{n \geqslant 1} \subseteq Y$ such that

$$
\left\|y_{n}\right\| \rightarrow \infty \text { and } \psi\left(y_{n}\right) \geqslant-M_{4} \text { for some } M_{4}>0 \text {, all } n \geqslant 1 \text {. }
$$

We have

$$
-M_{4} \leqslant \psi\left(y_{n}\right) \leqslant \varphi\left(y_{n}\right)=\frac{1}{2} \tau\left(y_{n}\right)-\int_{\Omega} F\left(z, y_{n}\right) d z \text { for all } n \geqslant 1 .
$$

Let $v_{n}=\frac{y_{n}}{\left\|y_{n}\right\|}, n \geqslant 1$. Then $\left\|v_{n}\right\|=1, v_{n} \in Y$ for all $n \geqslant 1$. Recall that the space $Y$ is finite dimensional. So, we may assume that

$$
v_{n} \rightarrow v \text { in } H^{1}(\Omega), v \in Y \text { and }\|v\|=1 .
$$

From (53) we have

$$
-\frac{M_{4}}{\left\|y_{n}\right\|^{2}} \leqslant \frac{1}{2} \tau\left(v_{n}\right)-\int_{\Omega} \frac{F\left(z, y_{n}\right)}{\left\|y_{n}\right\|^{2}} d z .
$$

As before (see the proof of Proposition 51), we have that

$$
\left\{\frac{F\left(\cdot, y_{n}(\cdot)\right)}{\left\|y_{n}\right\|^{2}}\right\}_{n \geqslant 1} \subseteq L^{1}(\Omega) \text { is uniformly integrable. }
$$

Then the Dunford-Pettis theorem and hypotheses $H_{1}(i),(i i)$ imply that

$$
\frac{F\left(\cdot, y_{n}(\cdot)\right)}{\left\|y_{n}\right\|^{2}} \stackrel{w}{\longrightarrow} \frac{1}{2} \xi^{*} v^{2} \text { in } L^{1}(\Omega), \text { with } \hat{\lambda}_{m} \leqslant \xi^{*}(z) \leqslant \eta(z) \text { a.e. in } \Omega .
$$

If in (55) we pass to the limit as $n \rightarrow \infty$ and use (54) and (56), then

$$
0 \leqslant \frac{1}{2} \tau(v)-\frac{1}{2} \int_{\Omega} \xi^{*} v^{2} d z
$$

If $\xi^{*} \neq \hat{\lambda}_{m}$, then since $v \in Y$ (see (54)), from (56), (157) and Proposition 3(b) we reach a contradiction, that is, $0 \leqslant \frac{1}{2} \tau(v)-\frac{1}{2} \int_{\Omega} \xi^{*} v^{2} d z<0$. 
So, suppose that $\xi^{*} \equiv \hat{\lambda}_{m}$ (see (56) ). Then from (5) and (57) we have that $v \in E\left(\hat{\lambda}_{m}\right) \backslash\{0\}$ (see also (54)). The UCP implies that $v(z) \neq 0$ for a.a. $z \in \Omega$, and so $\left|y_{n}(z)\right| \rightarrow \infty$ for a.a. $z \in \Omega$.

By virtue of hypothesis $H_{1}($ iii $)$ we know that given any $\xi>0$, we can find $M_{5}=M_{5}(\xi)>0$ such that

$$
f(z, x) x-2 F(z, x) \leqslant-\xi \text { for a.a. } z \in \Omega, \text { all }|x| \geqslant M_{5} .
$$

We have

$$
\begin{aligned}
& \frac{d}{d x}\left(\frac{F(z, x)}{x^{2}}\right)=\frac{f(z, x) x-2 F(z, x)}{x^{3}} \\
& \leqslant-\frac{\xi}{x^{3}} \text { for a.a. } z \in \Omega, \text { all } x \geqslant M_{5}(\text { see }(\underline{58})), \\
& \Rightarrow \frac{F(z, x)}{x^{2}}-\frac{F(z, u)}{u^{2}} \leqslant \frac{\xi}{2}\left[\frac{1}{x^{2}}-\frac{1}{u^{2}}\right] \text { for a.a. } z \in \Omega, \text { all } x \geqslant u \geqslant M_{5} .
\end{aligned}
$$

Passing to the limit as $x \rightarrow+\infty$ and using hypothesis $H_{1}(i i)$, we obtain

$$
\frac{\hat{\lambda}_{m}}{2} u^{2}-F(z, u) \leqslant-\frac{\xi}{2} \text { for a.a. } z \in \Omega \text {, all } u \geqslant M_{5} .
$$

Since $\xi>0$ is arbitrary, we infer that

$$
\lim _{u \rightarrow+\infty}\left[\frac{\hat{\lambda}_{m}}{2} u^{2}-F(z, u)\right]=-\infty \text { uniformly for a.a. } z \in \Omega .
$$

From (53), we have

$$
\begin{aligned}
-M_{4} & \leqslant \frac{1}{2} \tau\left(y_{n}\right)-\int_{\Omega} F\left(z, y_{n}\right) d z \\
& \leqslant \frac{\hat{\lambda}_{m}}{2}\left\|y_{n}\right\|_{2}^{2}-\int_{\Omega} F\left(z, y_{n}\right) d z\left(\text { since } y_{n} \in Y, \text { see (15) }\right) \\
& =\int_{\Omega}\left[\frac{\hat{\lambda}_{m}}{2} y_{n}^{2}-F\left(z, y_{n}\right)\right] d z .
\end{aligned}
$$

From (59) and Fatou's lemma (since $\left|y_{n}(z)\right| \rightarrow+\infty$ for a.a. $z \in \Omega$ ), we have

$$
\int_{\Omega}\left[\frac{\hat{\lambda}_{m}}{2} y_{n}^{2}-F\left(z, y_{n}\right)\right] d z \rightarrow-\infty \text { as } n \rightarrow \infty .
$$

Comparing (60) and (61), we reach a contradiction. This proves the anticoercivity of the functional $\psi$.

Remark 2. Since $-\psi$ is coercive, it follows that the functional $\psi$ satisfies the $C$ condition.

Now we are ready for the "three solutions theorem" for problem (11).

Theorem 13. If hypotheses $H_{0}$ and $H_{1}$ hold, then problem (11) has at least three nontrivial solutions,

$$
u_{0} \in \operatorname{int} C_{+}, v_{0} \in-i n t C_{+} \text {and } y_{0} \in C^{1}(\bar{\Omega}) .
$$


Proof. From Proposition 9 we already have two nontrivial constant sign solutions

$$
u_{0} \in \operatorname{int} C_{+} \text {and } v_{0} \in-\operatorname{int} C_{+} .
$$

From the proof of Proposition 9, we know that:

(i) $u_{0}$ is a critical point of mountain pass type for the functional $\hat{\varphi}_{+}$;

(ii) $v_{0}$ is a critical point of mountain pass type for the functional $\hat{\varphi}_{-}$.

Hence we have

$$
C_{1}\left(\hat{\varphi}_{+}, u_{0}\right) \neq 0 \text { and } C_{1}\left(\hat{\varphi}_{-}, v_{0}\right) \neq 0 \text { (see Chang [8]). }
$$

Note that

$$
\left.\hat{\varphi}_{+}\right|_{C_{+}}=\left.\varphi\right|_{C_{+}} \text {and }\left.\hat{\varphi}_{-}\right|_{-C_{+}}=\left.\varphi\right|_{-C_{+}} .
$$

From Chang [8, p. 14] (see also Bartsch [3]), we have

$$
C_{k}\left(\hat{\varphi}_{+}, u_{0}\right)=C_{k}\left(\left.\hat{\varphi}_{+}\right|_{C^{1}(\bar{\Omega})}, u_{0}\right) \text { and } C_{k}\left(\varphi, u_{0}\right)=C_{k}\left(\left.\varphi\right|_{C^{1}(\bar{\Omega})}, u_{0}\right) \text { for all } k \geqslant 0,
$$

(65) $C_{k}\left(\hat{\varphi}_{-}, v_{0}\right)=C_{k}\left(\left.\hat{\varphi}_{-}\right|_{C^{1}(\bar{\Omega})}, v_{0}\right)$ and $C_{k}\left(\varphi, v_{0}\right)=C_{k}\left(\left.\varphi\right|_{C^{1}(\bar{\Omega})}, v_{0}\right)$ for all $k \geqslant 0$.

From relations (62)- 65 and since $u_{0} \in \operatorname{int} C_{+}, v_{0} \in-\operatorname{int} C_{+}$, it follows that

$$
C_{1}\left(\varphi, u_{0}\right) \neq 0 \text { and } C_{1}\left(\varphi, v_{0}\right) \neq 0 \text {. }
$$

If $\bar{u}_{0}=p_{Y}\left(u_{0}\right), \bar{v}_{0}=p_{Y}\left(v_{0}\right)\left(p_{Y}\right.$ being the orthogonal projection of $H^{1}(\Omega)$ onto $Y)$, then from Liu \& Li [20], we have

$$
\begin{aligned}
& C_{k}\left(\varphi, u_{0}\right)=C_{k}\left(\psi, \bar{u}_{0}\right) \text { and } C_{k}\left(\varphi, v_{0}\right)=C_{k}\left(\psi, \bar{v}_{0}\right) \text { for all } k \geqslant 0, \\
\Rightarrow \quad & C_{1}\left(\psi, \bar{u}_{0}\right) \neq 0 \text { and } C_{1}\left(\psi, \bar{v}_{0}\right) \neq 0(\text { see }(\underline{66})) .
\end{aligned}
$$

From Proposition 7 we have

$$
\begin{aligned}
C_{k}(\varphi, 0) & =\delta_{k, 0} \mathbb{Z} \text { for all } k \geqslant 0, \\
\Rightarrow \quad C_{k}(\psi, 0) & =\delta_{k, 0} \mathbb{Z} \text { for all } k \geqslant 0(\text { see }[20]) .
\end{aligned}
$$

From Proposition 12, we have that $\psi$ is anticoercive. Also, it is continuous (recall that the space $Y$ is finite dimensional). Hence by the Weierstrass theorem we can find $\bar{y}_{0} \in Y$ such that $\psi\left(\bar{y}_{0}\right)=\sup _{Y} \psi$. Then we have

$$
C_{k}\left(\psi, \bar{y}_{0}\right)=\delta_{k, d_{m}} \mathbb{Z} \text { for all } k \geqslant 0 \text {, with } d_{m}=\operatorname{dim} Y \geqslant 2 \text {. }
$$

Comparing (69) with (67) and (68), we infer that

$$
\begin{aligned}
& \bar{y}_{0} \notin\left\{0, \bar{u}_{0}, v_{0}\right\}, \\
\Rightarrow & y_{0}=\bar{y}_{0}+\gamma_{0}\left(\bar{y}_{0}\right) \text { is the third distinct nontrivial solution. }
\end{aligned}
$$

As before, the regularity results of Wang [34] imply that $y_{0} \in C^{1}(\bar{\Omega})$.

\section{Four nONTRIVIAL SOLUtions}

By strengthening the regularity on $f(z, \cdot)$, we can improve Theorem 13 and produce four nontrivial solutions.

We start by computing the critical groups of the functional $\psi$ at infinity. To do this, we do not need any additional conditions on $f(z, \cdot)$. Our proof uses some ideas from Liu [18.

Proposition 14. If hypotheses $H_{0}$ and $H_{1}$ hold, then $C_{k}(\psi, \infty)=\delta_{k, d_{m}} \mathbb{Z}$ for all $k \geqslant 0$, with $d_{m}=\operatorname{dim} Y \geqslant 2$. 
Proof. As always, we assume that $K_{\psi}$ is finite (otherwise we already have an infinity of nontrivial solutions for problem (1) ).

Let $\hat{m}<\inf \psi\left(K_{\psi}\right)$. In what follows, for every $r>0$, we introduce the set

$$
C_{r}=\{y \in Y:\|y\| \geqslant r\} .
$$

Recall that $\psi$ is anticoercive (see Proposition 12). So, we can find $\lambda<\mu<\hat{m}$ and $0<\varrho<R$ such that

$$
C_{R} \subseteq \psi^{\lambda} \subseteq C_{\varrho} \subseteq \psi^{\mu}
$$

Then for the triples $\left(C_{R}, C_{\varrho}, Y\right)$ and $\left(\psi^{\lambda}, \psi^{\mu}, Y\right)$, we consider the corresponding long exact sequences of singular homology groups

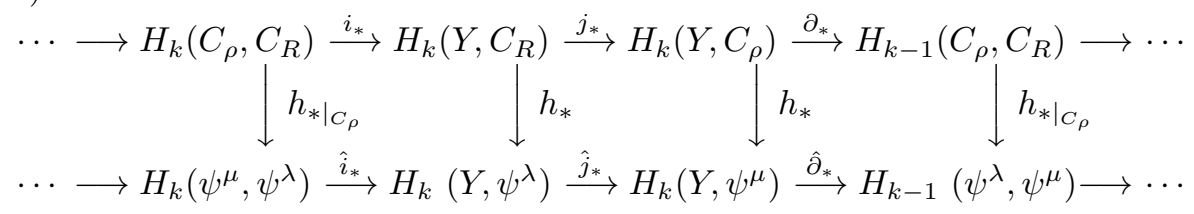

In (170) all squares are commutative (see Granas \& Dugundji [14, p. 377]), and $i_{*}, j_{*}, \hat{i}_{*}, \hat{j}_{*}, h_{*}$ are the group homomorphisms induced by the corresponding inclusion maps. Also, $\partial_{*}$ and $\hat{\partial}_{*}$ are the corresponding boundary homomorphisms. Recall that $\lambda<\mu<\hat{m}<\inf \psi\left(K_{\psi}\right)$. So, by the second deformation theorem (see, for example, Gasinski \& Papageorgiou [11, p. 628]), we have that $\psi^{\lambda}$ is a strong deformation retract of $\psi^{\mu}$. Hence

$$
H_{k}\left(\psi^{\mu}, \psi^{\lambda}\right)=0 \text { for all } k \geqslant 0 .
$$

Let $\chi: C_{\varrho} \rightarrow C_{R}$ be the map defined by

$$
\chi(u)= \begin{cases}R \frac{u}{\|u\|} & \text { if } \varrho \leqslant\|u\| \leqslant R \\ u & \text { if } R<\|u\| .\end{cases}
$$

Evidently $\chi$ is continuous and $\left.\chi\right|_{C_{R}}=\left.\operatorname{Id}\right|_{C_{R}}$. Therefore $C_{R}$ is a retract of $C_{\varrho}$. Consider the deformation $h:[0,1] \times C_{\varrho} \rightarrow Y$ defined by

$$
h(t, u)=(1-t) u+t R \frac{u}{\|u\|} \text { for all } t \in[0,1], \text { all } u \in C_{\varrho} .
$$

Then $h(0, u)=u$ and $h(1, u)=R \frac{u}{\|u\|} \in C_{R}$. So, we see that the set $C_{\varrho}$ is deformable onto $C_{R}$ over $Y$. Invoking Theorem 6.5 of Dugundji [9, p. 325], we infer that $C_{R}$ is a deformation retract of $C_{\varrho}$. Therefore from Granas \& Dugundji [14, p. 387], we have

$$
H_{k}\left(C_{\varrho}, C_{R}\right)=0 \text { for all } k \geqslant 0 .
$$

The exactness of the two long homology sequences in (170) and the rank theorem imply that

$$
\begin{aligned}
& 0=\operatorname{im} i_{*}=\operatorname{ker} j_{*} \text { and } \operatorname{im} j_{*}=\operatorname{ker} \partial_{*}=H_{k}\left(Y, C_{\varrho}\right)(\text { see (72) }), \\
& 0=\operatorname{im} \hat{i}_{*}=\operatorname{ker} \hat{j}_{*} \text { and } \operatorname{im} \hat{j}_{*}=\operatorname{ker} \hat{\partial}_{*}=H_{k}\left(Y, \psi^{\mu}\right)(\text { see (71) }) .
\end{aligned}
$$

So, we see that $j_{*}$ and $\hat{j}_{*}$ are group isomorphisms. Then from Granas \& Dugundji [14, p. 610], it follows that $h_{*}$ is an isomorphism. Hence

$$
\begin{aligned}
H_{k}\left(Y, C_{\varrho}\right) & =H_{k}\left(Y, \psi^{\mu}\right) \text { for all } k \geqslant 0, \\
\Rightarrow \quad H_{k}\left(Y, C_{\varrho}\right) & =C_{k}(\psi, \infty) \text { for all } k \geqslant 0(\text { recall } \mu<\hat{m}) .
\end{aligned}
$$


Using the radial retraction and Theorem 6.5 of Dugundji [9, p. 325], we see that $\partial B_{\varrho}^{Y}=\{y \in Y:\|y\|=\varrho\}$ is a deformation retract of $C_{\varrho}$. Therefore

$$
\begin{aligned}
& H_{k}\left(Y, C_{\varrho}\right)=H_{k}\left(Y, \partial B_{\varrho}\right) \text { for all } k \geqslant 0, \\
\Rightarrow \quad & H_{k}\left(Y, C_{\varrho}\right)=\delta_{k, d_{m}} \mathbb{Z} \text { for all } k \geqslant 0(\text { see Maunder [21, p. 121]), } \\
\Rightarrow \quad & C_{k}(\psi, \infty)=\delta_{k, d_{m}} \mathbb{Z} \text { for all } k \geqslant 0(\text { see }(73)) .
\end{aligned}
$$

We introduce the new stronger conditions on the reaction $f(z, x)$.

$H_{2}: f: \Omega \times \mathbb{R} \rightarrow \mathbb{R}$ is a measurable function such that for a.a. $z \in \Omega, f(z, 0)=0$, $f(z, \cdot) \in C^{1}(\mathbb{R})$ and

(i) there exist an integer $m \geqslant \max \left\{m_{0}, 2\right\}$ and a function $\eta \in L^{\infty}(\Omega)_{+}$such that

$$
\begin{aligned}
& \eta(z) \leqslant \hat{\lambda}_{m+1} \text { a.e. in } \Omega, \eta \neq \hat{\lambda}_{m+1}, \\
& \left|f_{x}^{\prime}(z, x)\right| \leqslant \eta(z) \text { for a.a. } z \in \Omega, \text { all } x \in \mathbb{R} ;
\end{aligned}
$$

(ii) $\hat{\lambda}_{m} \leqslant \liminf _{x \rightarrow \pm \infty} \frac{f(z, x)}{x}$ uniformly for a.a. $z \in \Omega$;

(iii) $\lim _{x \rightarrow \pm \infty}[f(z, x) x-2 F(z, x)]=-\infty$ uniformly for a.a. $z \in \Omega$;

(iv) there exists a function $\vartheta \in L^{\infty}(\Omega)+$ such that

$$
\begin{aligned}
& \vartheta(z) \leqslant \hat{\lambda}_{1} \text { a.e. in } \Omega, \vartheta \neq \hat{\lambda}_{1}, \\
& \limsup _{x \rightarrow 0} \frac{2 F(z, x)}{x^{2}} \leqslant \vartheta(z) \text { uniformly for a.a. } z \in \Omega .
\end{aligned}
$$

Remark 3. Note that hypothesis $H_{2}(i)$ and the mean value theorem imply that

$$
|f(z, x)| \leqslant \eta(z)|x| \text { for a.a. } z \in \Omega \text {, all } x \in \mathbb{R} \text {. }
$$

Also, for every $\varrho>0$, there exists $\xi_{\varrho}>0$ such that for a.a. $z \in \Omega, x \mapsto f(z, x)+\xi_{\varrho} x$ is nondecreasing on $[-\varrho, \varrho]$.

Example 2. The following function satisfies hypotheses $H_{2}$. As before, for the sake of simplicity we drop the $z$-dependence:

$$
f(x)= \begin{cases}\vartheta x+\frac{\xi x}{1+|x|} & \text { if }|x| \leqslant 1, \\ \lambda x+\frac{c}{x} & \text { if } 1<|x|,\end{cases}
$$

with $\vartheta<\hat{\lambda}_{1}, \lambda \in\left(\hat{\lambda}_{m}, \hat{\lambda}_{m+1}\right)$ for some integer $m \geqslant \max \left\{m_{0}, 2\right\}, \xi, c>0, \vartheta+\xi<$ $\hat{\lambda}_{m+1}$.

Theorem 15. If hypotheses $H_{0}$ and $H_{2}$ hold, then problem (1) admits at least four nontrivial solutions,

$$
u_{0} \in \operatorname{int} C_{+}, v_{0} \in-i n t C_{+} \text {and } y_{0}, \hat{y} \in C^{1}(\bar{\Omega}) .
$$

Proof. From Theorem 13 we already have three nontrivial solutions,

$$
u_{0} \in \operatorname{int} C_{+}, v_{0} \in-\operatorname{int} C_{+} \text {and } y_{0} \in C^{1}(\bar{\Omega}) .
$$

In this case $\varphi \in C^{2}\left(H^{1}(\Omega)\right)$. From the proof of Proposition 9 and since $\left.\hat{\varphi}_{+}\right|_{C_{+}}=$ $\left.\varphi\right|_{C_{+}},\left.\hat{\varphi}_{-}\right|_{-C_{+}}=\left.\varphi\right|_{-C_{+}}$, we see that $u_{0} \in \operatorname{int} C_{+}$and $v_{0} \in-\operatorname{int} C_{+}$are critical points of mountain pass type for $\varphi$, and so

$$
C_{k}\left(\varphi, u_{0}\right)=C_{k}\left(\varphi, v_{0}\right)=\delta_{k, 1} \mathbb{Z} \text { for all } k \geqslant 0 \text { (see Bartsch [3]). }
$$


From (69) we have that $C_{k}\left(\psi, \bar{y}_{0}\right)=\delta_{k, d_{m}} \mathbb{Z}$ for all $k \geqslant 0$ (recall that $\bar{y}_{0}=p_{Y}\left(y_{0}\right)$ ). From Liu \& Li [20] we know that $C_{k}\left(\varphi, y_{0}\right)=C_{k}\left(\psi, \bar{y}_{0}\right)$. Therefore

$$
C_{k}\left(\varphi, y_{0}\right)=\delta_{k, d_{m}} \mathbb{Z} \text { for all } k \geqslant 0 \text {. }
$$

Also, we have

$$
\begin{gathered}
C_{k}(\varphi, 0)=\delta_{k, 0} \mathbb{Z} \text { for all } k \geqslant 0 \text { (see Proposition } 7 \text { ), } \\
C_{k}(\varphi, \infty)=\delta_{k, d_{m}} \mathbb{Z} \text { for all } k \geqslant 0 \text { (see Proposition 14). }
\end{gathered}
$$

Suppose that $K_{\varphi}=\left\{0, u_{0}, v_{0}, y_{0}\right\}$. Then from (74), (175), (76), (177) and the Morse relation (see (17)) with $t=-1$, we have

$$
\begin{aligned}
& (-1)^{0}+2(-1)^{1}+(-1)^{d_{m}}=(-1)^{d_{m}}, \\
\Rightarrow \quad & (-1)^{1}=0, \text { a contradiction. }
\end{aligned}
$$

So, we can find $\hat{y} \in K_{\varphi} \backslash\left\{0, u_{0}, v_{0}, y_{0}\right\}$. Evidently $\hat{y}$ is the fourth nontrivial solution of (11), and as before, using the regularity results of Wang [34, we have that $\hat{y} \in$ $C^{1}(\bar{\Omega})$.

\section{Coercive problems}

In this section, we study what happens when the limit in hypothesis $H_{1}(i i i)$ is $+\infty$. In this case, the geometry of the problem changes since, as we will see, the reduced functional $\psi$ is coercive. We can still prove a multiplicity theorem producing at least two nontrivial solutions.

The new hypotheses on the reaction $f(z, x)$ are the following:

$H_{3}: f: \Omega \times \mathbb{R} \rightarrow \mathbb{R}$ is a Carathéodory function such that $f(z, 0)=0$ for a.a. $z \in \Omega$ and

(i) there exist an integer $m \geqslant \max \left\{m_{0}, 2\right\}$ and a function $\eta \in L^{\infty}(\Omega)+$ such that

$$
\begin{aligned}
& \eta(z) \leqslant \hat{\lambda}_{m+1} \text { a.e. in } \Omega, \eta \neq \hat{\lambda}_{m+1}, \\
& (f(z, x)-f(z, y))(x-y) \leqslant \eta(z)(x-y)^{2} \text { for a.a. } z \in \Omega, \text { all } x, y \in \mathbb{R} ;
\end{aligned}
$$

(ii) $\hat{\lambda}_{m} \leqslant \liminf _{x \rightarrow \pm \infty} \frac{f(z, x)}{x}$ uniformly for a.a. $z \in \Omega$;

(iii) $\lim _{x \rightarrow \pm \infty}[f(z, x) x-2 F(z, x)]=+\infty$ uniformly for a.a. $z \in \Omega$;

(iv) there exist an integer $1<k<m-1$, a function $\eta_{0} \in L^{\infty}(\Omega)$ and $\delta_{0}>0$ such that

$$
\begin{aligned}
& \hat{\lambda}_{k} \leqslant \eta_{0}(z) \text { a.e. in } \Omega, \eta_{0} \neq \hat{\lambda}_{k}, \\
& \eta_{0}(z) \leqslant \liminf _{x \rightarrow 0} \frac{2 F(z, x)}{x^{2}} \text { uniformly for a.a. } z \in \Omega, \\
& F(z, x) \leqslant \frac{\hat{\lambda}_{k+1}}{2} x^{2} \text { for a.a. } z \in \Omega, \text { all }|x| \leqslant \delta_{0} .
\end{aligned}
$$

Remark 4. Note that compared to hypothesis $H_{1}(i v)$, now hypothesis $H_{3}(i v)$ implies a different geometry for which $u=0$ is no longer a local minimizer of the energy functional. 
Example 3. The following function satisfies hypotheses $H_{3}$. As always, we drop the $z$-dependence:

$$
f(x)= \begin{cases}\beta x+\xi|x|^{p-2} x & \text { if }|x| \leqslant 1 \\ \lambda x-\frac{c}{x} & \text { if } 1<|x|\end{cases}
$$

with $\beta \in\left(\hat{\lambda}_{k}, \hat{\lambda}_{k+1}\right), \xi, c>0, \lambda \in\left(\hat{\lambda}_{m}, \hat{\lambda}_{m+1}\right), m \geqslant \max \left\{m_{0}, 2\right\}, m>k+1, \beta+$ $\xi<\hat{\lambda}_{m+1}$ and $\beta+\xi=\lambda-c$.

Again we consider the following orthogonal direct sum decomposition of the space $H^{1}(\Omega)$ :

$$
H^{1}(\Omega)=Y \oplus \hat{H} \text { with } Y=\bigoplus_{i=1}^{m} E\left(\hat{\lambda}_{i}\right), \hat{H}=\overline{\bigoplus_{i \geqslant m+1} E\left(\hat{\lambda}_{i}\right)} .
$$

As before (see Proposition 10 and its proof), using the reduction method, we produce a continuous map $\gamma: Y \rightarrow \hat{H}$ such that

$$
\begin{aligned}
& \quad \psi(y)=\varphi(y+\gamma(y))=\inf [\varphi(y+\hat{u}): \hat{u} \in \hat{H}] \\
& \text { and } \quad \psi \in C^{1}(Y) \text { (see Proposition 11). }
\end{aligned}
$$

Reasoning as in the proof of Proposition 8, we obtain:

Proposition 16. If hypotheses $\mathrm{H}_{0}$ and $\mathrm{H}_{3}$ hold, then the reduced functional $\psi$ is coercive.

Using this proposition, we obtain the following multiplicity theorem.

Theorem 17. If hypotheses $H_{0}$ and $H_{3}$ hold, then problem (1) admits at least two nontrivial solutions,

$$
u_{0}, \hat{u} \in C^{1}(\bar{\Omega}) .
$$

Proof. From Proposition 16, we know that $\psi$ is coercive, hence it is bounded from below.

We consider the following orthogonal direct sum decomposition of $Y$ :

$$
Y=\bar{H}_{k} \oplus V,
$$

where $\bar{H}_{k}=\bigoplus_{i=1}^{k} E\left(\hat{\lambda}_{i}\right)$ and $V=\bigoplus_{i=k+1}^{m} E\left(\hat{\lambda}_{i}\right)($ recall $k<m-1)$.

By virtue of hypotheses $H_{3}(i),(i v)$, given $\varepsilon>0$ and $r>2$, we can find $c_{3}=$ $c_{3}(\varepsilon, r)>0$ such that

$$
F(z, x) \geqslant \frac{1}{2}\left(\eta_{0}(z)-\varepsilon\right) x^{2}-c_{3}|x|^{r} \text { for a.a. } z \in \Omega, \text { all } x \in \mathbb{R} .
$$

Then for all $u \in \bar{H}_{k}$ we have

$$
\begin{aligned}
\psi(u) & =\varphi(u+\gamma(u)) \\
& \leqslant \varphi(u) \\
& =\frac{1}{2} \tau(u)-\int_{\Omega} F(x, u) d z \\
& \leqslant \frac{1}{2} \tau(u)-\frac{1}{2} \int_{\Omega} \eta_{0} u^{2} d z+\frac{\varepsilon}{2}\|u\|^{2}+c_{4}\|u\|^{r} \text { for some } c_{4}>0 \text { (see (78)) } \\
& \left.\leqslant \frac{-\hat{\xi}_{2}+\varepsilon}{2}\|u\|^{2}+c_{4}\|u\|^{r} \text { (see Proposition } 3(b)\right) .
\end{aligned}
$$


Choosing $\varepsilon \in\left(0, \hat{\xi}_{2}\right)$ and since $r>2$, from (179) we see that we can find $\varrho_{2} \in(0,1)$ small such that

$$
\psi(u) \leqslant 0 \text { for all } u \in \bar{H}_{k}, \text { with }\|u\| \leqslant \varrho_{2} .
$$

Moreover, from (79) it is clear that

$$
\inf _{Y} \psi<0 .
$$

Since $V$ is finite dimensional, all norms are equivalent, and so we can find $\delta_{1}>0$ such that

$$
u \in V \text { with }\|u\| \leqslant \delta_{1} \Rightarrow|u(z)| \leqslant \delta_{0} \text { for all } z \in \bar{\Omega} .
$$

Here $\delta_{0}>0$ is as postulated by hypothesis $H_{3}(i v)$. Exploiting the continuity of the map $\gamma(\cdot)$ and since $\gamma(0)=0$, we can find $\varrho_{3}=\varrho_{3}\left(\delta_{1}\right)>0$ such that

$$
v \in V \text { with }\|v\| \leqslant \varrho_{3} \Rightarrow\|v+\gamma(v)\| \leqslant \delta_{1} .
$$

Then from (82) and (83), we see that if $v \in V$ with $\|v\| \leqslant \varrho_{3}$, then

$$
\begin{aligned}
& \psi(v)=\varphi(v+\gamma(v)) \\
& =\frac{1}{2} \tau(v+\gamma(v))-\int_{\Omega} F(z, v+\gamma(v)) d z \\
& \geqslant \frac{1}{2} \tau(v+\gamma(v))-\frac{\hat{\lambda}_{k+1}}{2}\|v+\gamma(v)\|_{2}^{2}\left(\text { see (82), (83) } \text { and } H_{3}(i v)\right) \\
& \geqslant 0\left(\text { since } v+\gamma(v) \in \overline{\bigoplus_{i \geqslant k+1} E\left(\hat{\lambda}_{i}\right)} \text {; see (5) }\right) \text {. }
\end{aligned}
$$

From (80), (81) and (84), we see that we can apply Proposition 4 and produce two nontrivial critical points $v_{0}, \hat{v} \in V$ of $\psi$. Then $u_{0}=v_{0}+\gamma\left(v_{0}\right)$ and $\hat{u}=\hat{v}+\gamma(\hat{v})$ are critical points of $\varphi$ (see [20]), hence solutions of (11). Moreover, as before the regularity theory (see Wang [34]) implies that $u_{0}, \hat{u} \in C^{1}(\bar{\Omega})$.

\section{Anticoercive problems}

Keeping the same geometry near the origin, we see what happens when the asymptotic condition $\pm \infty$ makes the reduced functional $\psi$ anticoercive. Again we can prove a multiplicity theorem producing two nontrivial solutions. As in Section 5 our main tool is Proposition 4 (the local linking multiplicity result).

In this section we assume that 0 is in the spectrum of $-\Delta+\beta$ (denoted by $\sigma(-\Delta+\beta))$ and this spectrum has also negative elements. In what follows by $\hat{\lambda}_{m}>0$ (resp. $\hat{\lambda}_{k}<0$ ) we denote the first strictly positive (resp. strictly negative) eigenvalue of $-\Delta+\beta$.

The conditions on the reaction term $f(z, x)$ are the following:

$H_{4}: f: \Omega \times \mathbb{R} \rightarrow \mathbb{R}$ is a Carathéodory function such that $f(z, 0)=0$ for a.a. $z \in \Omega$ and

(i) there exist a set $B \subseteq \Omega$ with $|B|_{N}>0$ and $\xi \in L^{1}(\Omega)$ such that

$$
\begin{aligned}
& F(z, x) \rightarrow+\infty \text { for a.a. } z \in \Omega, \text { as } x \rightarrow \pm \infty, \\
& F(z, x) \geqslant \xi(z) \text { for a.a. } z \in \Omega, \text { all } x \in \mathbb{R} ;
\end{aligned}
$$


(ii) there exists a function $\eta \in L^{\infty}(\Omega)+$ such that

$$
\begin{aligned}
& \eta(z) \leqslant \hat{\lambda}_{m} \text { for a.a. } z \in \Omega, \eta \neq \hat{\lambda}_{m}, \\
& (f(z, x)-f(z, y))(x-y) \leqslant \eta(z)(x-y)^{2} \text { for a.a. } z \in \Omega, \text { all } x, y \in \mathbb{R} ;
\end{aligned}
$$

(iii) there exist a function $\eta_{0} \in L^{\infty}(\Omega)$ and $\delta_{0}>0$ such that

$$
\begin{aligned}
& \eta_{0}(z) \leqslant 0 \text { a.e. in } \Omega, \eta_{0} \neq 0, \\
& \limsup _{x \rightarrow 0} \frac{2 F(z, x)}{x^{2}} \leqslant \eta_{0}(z) \text { uniformly for a.a. } z \in \Omega, \\
& \frac{\hat{\lambda}_{k}}{2} x^{2} \leqslant F(z, x) \text { for a.a. } z \in \Omega, \text { all }|x| \leqslant \delta_{0} .
\end{aligned}
$$

Remark 5. We see that the condition near zero (see $H_{4}(i v)$ ) is similar to that in $H_{3}(i v)$. In particular, both imply that the origin cannot be a local minimizer of the energy functional. So, the geometry of the problem differs from that assumed in Sections 3 and 4 .

Example 4. The following function satisfies hypotheses $H_{4}$. Again, we drop the $z$-dependence:

$$
f(x)= \begin{cases}\eta_{0} x & \text { if }|x| \leqslant 1, \\ \lambda x-\beta & \text { if } 1<|x|,\end{cases}
$$

with $\eta_{0} \in\left(\hat{\lambda}_{k}, 0\right), \lambda \in\left(0, \hat{\lambda}_{m}\right)$ and $\beta=\lambda-\eta_{0}$.

We introduce the following subspaces of $H^{1}(\Omega)$ :

$$
\bar{H}=\bigoplus_{i=1}^{k} E\left(\hat{\lambda}_{i}\right), E=E(0), Y=\bar{H} \oplus E \text { and } \hat{H}=Y^{\perp}
$$

Reasoning as in the proof of Proposition 10, we obtain:

Proposition 18. If hypotheses $H_{0}$ and $H_{4}$ hold, then there exists a continuous map $\hat{\gamma}: Y \rightarrow \hat{H}$ such that

$$
\varphi(y+\hat{\gamma}(y))=\inf [\varphi(y+\hat{u}): \hat{u} \in \hat{H}] .
$$

We set $\psi(y)=\varphi(y+\hat{\gamma}(y))$ for all $y \in Y$, and as in Proposition 11, we show that:

Proposition 19. If hypotheses $H_{0}$ and $H_{4}$ hold, then $\psi \in C^{1}(Y)$.

Using the chain rule, we have

$$
\psi^{\prime}(y)=p_{Y *} \varphi^{\prime}(y+\hat{\gamma}(y)) \text { for all } y \in Y,
$$

with $p_{Y^{*}}$ being the orthogonal projection onto $Y^{*}$.

Also, we introduce the functional

$$
\vartheta(y)=-\psi(y) \text { for all } y \in Y .
$$

Proposition 20. If hypotheses $H_{0}$ and $H_{4}$ hold, then there exists $\varrho>0$ such that

$$
\begin{aligned}
& \vartheta(y) \leqslant 0 \text { for all } y \in \bar{H} \text { with }\|y\| \leqslant \varrho, \\
& \vartheta(y) \geqslant 0 \text { for all } y \in E \text { with }\|y\| \leqslant \varrho .
\end{aligned}
$$


Proof. By virtue of hypotheses $H_{4}(i i),($ iii $)$, given $\varepsilon>0$ and $r>2$, we can find $c_{4}=c_{4}(\varepsilon, r)>0$ such that

$$
F(z, x) \leqslant \frac{\eta_{0}(z)+\varepsilon}{2} x^{2}+c_{4}|x|^{r} \text { for a.a. } z \in \Omega, \text { all } x \in \mathbb{R}
$$

Then for all $y \in E$, we have

$$
\begin{aligned}
\vartheta(y)=-\psi(y)= & -\varphi(y+\hat{\gamma}(y)) \\
= & -\frac{1}{2} \tau(y+\hat{\gamma}(y))+\int_{\Omega} F(z, y+\hat{\gamma}(y)) d z \\
\leqslant & -\frac{1}{2} \tau(y+\hat{\gamma}(y))+\frac{1}{2} \int_{\Omega} \eta_{0}(y+\hat{\gamma}(y))^{2} d z+\frac{\varepsilon}{2}\|y+\hat{\gamma}(y)\|^{2} \\
& +c_{5}\|y+\hat{\gamma}(y)\|^{r}\left(\text { for some } c_{5}>0(\text { see [850) })\right) \\
\leqslant & \left.-\frac{\xi_{1}-\varepsilon}{2}\|y+\hat{\gamma}(y)\|^{2}+c_{5}\|y+\hat{\gamma}(y)\|^{r} \text { (see Proposition } 3(a)\right) .
\end{aligned}
$$

From hypothesis $H_{4}(i i)$, we have

$$
F(z, x) \leqslant \frac{\eta(z)}{2} x^{2} \text { for a.a. } z \in \Omega, \text { all } x \in \mathbb{R}
$$

For every $\hat{u} \in \hat{H}$, we have

$$
\begin{aligned}
\varphi(\hat{u}) & =\frac{1}{2} \tau(\hat{u})-\int_{\Omega} F(z, \hat{u}) d z \\
& \geqslant \frac{1}{2} \tau(\hat{u})-\frac{1}{2} \int_{\Omega} \eta \hat{u}^{2} d z(\text { see }(\underline{87})) \\
& \geqslant \frac{\xi_{1}}{2}\|\hat{u}\|^{2} \quad(\text { see Proposition } \underline{3}(a)), \\
\Rightarrow \quad & \quad \inf _{\varphi} \varphi=0, \\
\Rightarrow \quad & \hat{\gamma}(0)=0 .
\end{aligned}
$$

Recalling that $\hat{\gamma}$ is continuous (see Proposition [18), from (86) and (88) it follows that we can find $\varrho_{1} \in(0,1)$ small such that

$$
\vartheta(y) \leqslant 0 \text { for all } y \in E \text { with }\|y\| \leqslant \varrho_{1} .
$$

On the other hand, since $\bar{H}$ is finite dimensional, all norms are equivalent, and so we can find $c_{6}>0$ such that

$$
\|y\|_{\infty} \leqslant c_{6}\|y\| \text { for all } y \in \bar{H} \text {. }
$$

Let $\delta_{0}>0$ be as postulated by hypothesis $H_{4}(i i i)$. Then for $\varrho_{2}=\frac{\delta_{0}}{c_{6}}>0$ we have

$$
\left.y \in \bar{H},\|y\| \leqslant \varrho_{2} \Rightarrow|y(z)| \leqslant \delta_{0} \text { for all } z \in \bar{\Omega} \text { (see (90) }\right) \text {. }
$$


Then by virtue of hypothesis $H_{4}(i i i)$ we have

$$
\begin{aligned}
F(z, y(z)) & \geqslant \frac{\hat{\lambda}_{k}}{2} y(z)^{2} \text { for a.a. } z \in \Omega, \\
\Rightarrow \vartheta(y)=-\psi(y) & \geqslant-\varphi(y) \\
& \geqslant-\frac{1}{2} \tau(y)+\int_{\Omega} F(z, y) d z \\
& \geqslant-\frac{1}{2} \tau(y)+\frac{\hat{\lambda}_{k}}{2}\|y\|_{2}^{2} \text { (see (91)) } \\
& \left.\geqslant 0, \text { for all } y \in \hat{H} \text { with }\|y\| \leqslant \varrho_{2} \text { (see (50) }\right) .
\end{aligned}
$$

Choosing $\varrho=\min \left\{\varrho_{1}, \varrho_{2}\right\}$ from (89) and (92), we infer the result of the proposition (that is, we have local linking for $\vartheta$ ).

In order to use Proposition 4, we need to check that the functional $\vartheta$ is bounded below and satisfies the $C$-condition. To this end, we will need the following simple lemma.

Lemma 21. For every $\varepsilon>0$, there exists $\xi_{\varepsilon}>0$ such that

$$
\left|\left\{z \in \Omega:|y(z)|<\xi_{\varepsilon}\|y\|\right\}\right|_{N}<\varepsilon \text { for all } y \in E .
$$

Proof. We argue by contradiction. So, suppose we can find $\varepsilon>0$ and $\left\{y_{n}\right\}_{n \geqslant 1} \subseteq E$ such that $\left|C_{n}\right|_{N} \geqslant \varepsilon$ for all $n \geqslant 1$, where $C_{n}=\left\{z \in \Omega:\left|y_{n}(z)\right|<\frac{1}{n}\left\|y_{n}\right\|\right\}$. Let $v_{n}=\frac{y_{n}}{\left\|y_{n}\right\|}, n \geqslant 1$. Then $\left\|v_{n}\right\|=1, v_{n} \in E=E(0)$ for all $n \geqslant 1$. Since $E$ is finite dimensional, by passing to a suitable subsequence if necessary, we may assume that

$$
v_{n} \rightarrow v \text { in } H^{1}(\Omega) \text {, hence }\|v\|=1, v \in E .
$$

We have

$$
\begin{aligned}
& \limsup _{n \rightarrow \infty} C_{n} \subseteq C_{0}=\{z \in \Omega: y(z)=0\}, \\
\Rightarrow \quad & \varepsilon \leqslant \limsup _{n \rightarrow \infty}\left|C_{n}\right|_{N} \leqslant\left|\limsup _{n \rightarrow \infty} C_{n}\right|_{N} \leqslant\left|C_{0}\right|_{N} .
\end{aligned}
$$

From (93) we know that $y \neq 0, y \in E=E(0)$. So, by the UCP, we have $y(z) \neq 0$ a.e. in $\Omega$, which contradicts (94).

Using this lemma, we can establish the desired properties of $\vartheta$ in order to eventually apply Proposition 4 .

Proposition 22. If hypotheses $H_{0}$ and $H_{4}$ hold, then $\vartheta$ is coercive.

Proof. From the definition of $\vartheta$, we have

$$
\vartheta=-\psi \geqslant-\left.\varphi\right|_{Y} \text {. }
$$

So, to prove the proposition, it suffices to show that $-\left.\varphi\right|_{Y}$ is coercive. We argue indirectly. So, suppose that $-\left.\varphi\right|_{Y}$ is not coercive. Then we can find $M_{6}>0$ and $\left\{y_{n}\right\}_{n \geqslant 1} \subseteq Y$ such that

$$
-M_{6} \leqslant \varphi\left(y_{n}\right) \text { for all } n \geqslant 1 \text { and }\left\|y_{n}\right\| \rightarrow \infty .
$$

From hypothesis $H_{4}(i)$ and Tang \& Wu [31, we know that given $\varepsilon>0$, we can find $C_{\varepsilon} \subseteq B$ measurable such that

$$
\left|B \backslash C_{\varepsilon}\right|_{N}<\varepsilon \text { and } F(z, x) \rightarrow+\infty \text { uniformly for a.a. } z \in C_{\varepsilon} \text { as } x \rightarrow \pm \infty,
$$


and also there exist functions $h \in L^{1}(B)_{+}$and $g \in C(\mathbb{R})$ such that

$$
g \geqslant 0, g \text { is subadditive, }
$$

$$
F(z, x) \geqslant g(x)-h(z) \text { for a.a. } z \in C_{\varepsilon} \text {, all } x \in \mathbb{R},
$$

$g$ is coercive (that is, $g(x) \rightarrow+\infty$ as $x \rightarrow \pm \infty$ ),

From (95) we have

$$
\begin{aligned}
-M_{6} \leqslant \varphi\left(y_{n}\right)= & \frac{1}{2} \tau\left(y_{n}\right)-\int_{\Omega} F\left(z, y_{n}\right) d z \\
= & \frac{1}{2} \tau\left(y_{n}\right)-\int_{C_{\varepsilon}} F\left(z, y_{n}\right) d z-\int_{\Omega \backslash C_{\varepsilon}} F\left(z, y_{n}\right) d z \\
\leqslant & \left.\frac{1}{2} \tau\left(y_{n}\right)-\int_{C_{\varepsilon}} g\left(y_{n}\right) d z+c_{7} \text { for some } c_{7}>0 \text { (see (98) and } H_{4}(i)\right) \\
\leqslant & -\frac{c_{8}}{2}\left\|\bar{y}_{n}\right\|^{2}+c_{7}, \text { for some } c_{8}>0 \text { with } y_{n}=\bar{y}_{n}+y_{n}^{0}, \bar{y}_{n} \in \bar{H}, y_{n}^{0} \in E \\
& (\text { since } g \geqslant 0, \text { see (97) }),
\end{aligned}
$$

$\Rightarrow\left\{\bar{y}_{n}\right\}_{n \geqslant 1} \subseteq \bar{H} \subseteq H^{1}(\Omega)$ is bounded.

We have

$$
\left\|y_{n}\right\| \leqslant\left\|\bar{y}_{n}\right\|+\left\|y_{n}^{0}\right\| \text { for all } n \geqslant 1 \text { and }\left\|y_{n}^{0}\right\| \rightarrow+\infty \text { as } n \rightarrow \infty,
$$

We fix $\delta>0$. By virtue of Lemma 21, we can find $\xi_{\delta}>0$ such that

$$
\left|\left\{z \in \Omega:\left|y^{0}(z)\right|<\xi_{\delta}\left\|y^{0}\right\|\right\}\right|_{N}<\delta \text { for all } y^{0} \in E .
$$

Let $D_{n}=\left\{z \in \Omega:\left|y_{n}^{0}(z)\right| \geqslant \xi_{\delta}\left\|y_{n}^{0}\right\|\right\}$. Then $\left|\Omega \backslash D_{n}\right|_{N}<\delta$. Since $\bar{H}$ is finite dimensional and $\left\{\bar{y}_{n}\right\}_{n \geqslant 1}$ is bounded, we can find $c_{9}>0$ such that

$$
\left\|\bar{y}_{n}\right\|_{\infty} \leqslant c_{9} \text { for all } n \geqslant 1 .
$$

From (99) we know that $g$ is coercive. So, given $\mu>0$, we can find $M_{7}=M_{7}(\mu)>0$ such that

$$
g(x) \geqslant \mu \text { for all }|x| \geqslant M_{7} .
$$

Let $\Gamma_{n}=\left\{z \in \Omega:\left|y_{n}(z)\right| \geqslant M_{7}\right\}$. Then

$$
\begin{aligned}
& \left|y_{n}(z)\right| \geqslant\left|y_{n}^{0}(z)\right|-\left|\bar{y}_{n}(z)\right| \geqslant \xi_{\delta}\left\|y_{n}^{0}\right\|-c_{9} \text { for a.a. } z \in D_{n}, \\
& \text { all } n \geqslant 1(\text { see (102) }), \\
\Rightarrow & \left.\left|y_{n}(z)\right| \geqslant M_{7} \text { for all } z \in D_{n}, \text { all } n \geqslant n_{0} \text { (see (101) }\right), \\
\Rightarrow \quad & D_{n} \subseteq \Gamma_{n} \text { for all } n \geqslant n_{0} .
\end{aligned}
$$

We have

$$
\begin{aligned}
\int_{C_{\varepsilon}} g\left(y_{n}\right) d z & =\int_{C_{\varepsilon} \cap \Gamma_{n}} g\left(y_{n}\right) d z+\int_{C_{\varepsilon} \backslash \Gamma_{n}} g\left(y_{n}\right) d z \\
& \left.\geqslant \mu\left|C_{\varepsilon} \cap \Gamma_{n}\right|_{N} \text { (see (103) and recall } g \geqslant 0 \text { (see (197) }\right) \text { ). }
\end{aligned}
$$


Also,

$$
\begin{aligned}
&\left|C_{\varepsilon} \cap D_{n}\right|_{N}=\left|C_{\varepsilon}\right|_{N}-\left|C_{\varepsilon} \backslash D_{n}\right|_{N} \\
& \geqslant\left|C_{\varepsilon}\right|_{N}-\left|\Omega \backslash D_{n}\right|_{N} \\
& \geqslant|B|_{N}-\left|B \backslash C_{\varepsilon}\right|_{N}-\delta \\
& \geqslant|B|_{N}-\varepsilon-\delta>0 \text { for } \varepsilon, \delta>0 \text { small, } \\
& \Rightarrow\left|C_{\varepsilon} \cap \Gamma_{n}\right|_{N}>0 \text { for all } n \geqslant n_{0}(\text { see (104) }) .
\end{aligned}
$$

Since $\mu>0$ is arbitrary, from (105) and (106) it follows that

$$
\lim _{n \rightarrow \infty} \int_{C_{\varepsilon}} g\left(y_{n}\right) d z=+\infty
$$

Recall that

$$
\begin{aligned}
-M_{6} \leqslant \varphi\left(y_{n}\right) & \leqslant \frac{1}{2} \tau\left(y_{n}\right)-\int_{\Omega} g\left(y_{n}\right) d z+c_{7} \\
& \leqslant-\int_{\Omega} g\left(y_{n}\right) d z+c_{7}\left(\text { since } y_{n} \in Y(\text { see (5.5) })\right),
\end{aligned}
$$

which contradicts (107) above. This proves the coercivity of the functional $\vartheta$.

The coercivity of $\vartheta$ implies that $\vartheta$ satisfies the $C$-condition and it is bounded below. Moreover, from (86) and since $2<r$, we see that $\inf _{Y} \vartheta<0$. These facts and Proposition 20 permit the use of Proposition 4, and so we obtain the following multiplicity theorem for problem (11).

Theorem 23. If hypotheses $H_{0}$ and $H_{4}$ hold, then problem (11) admits at least two nontrivial solutions,

$$
u_{0}, \hat{u} \in C^{1}(\bar{\Omega}) .
$$

\section{REDUCTION ON AN INFINITE DIMENSIONAL SPACE}

In this section we examine what happens when the perturbed monotonicity condition in hypothesis $H_{4}(i)$ is reversed. Then the reduction method is implemented on an infinite dimensional subspace of $H^{1}(\Omega)$, and so the situation is more delicate.

The new hypotheses on the reaction $f(z, x)$ are the following:

$H_{5}: f: \Omega \times \mathbb{R} \rightarrow \mathbb{R}$ is a Carathéodory function such that $f(z, 0)=0$ for a.a. $z \in \Omega$ and

(i) $|f(z, x)| \leqslant a(z) x$ for a.a. $z \in \Omega$, all $x \in \mathbb{R}$ with $a \in L^{\infty}(\Omega)_{+}$;

(ii) there exist a function $\eta \in L^{\infty}(\Omega)$ and $M_{8}>0$ such that

$$
\begin{aligned}
& \eta(z) \geqslant \hat{\lambda}_{k} \text { for a.a. } z \in \Omega, \eta \neq \hat{\lambda}_{k}, \\
& (f(z, x)-f(z, y))(x-y) \geqslant \eta(z)(x-y)^{2} \text { for a.a. } z \in \Omega, \text { all } x, y \in \mathbb{R}, \\
& F(z, x) \leqslant 0 \text { for a.a. } z \in \Omega, \text { all }|x| \geqslant M_{8} ;
\end{aligned}
$$

(iv) $2 F(z, x)-f(z, x) x \rightarrow \pm \infty$ uniformly for a.a. $z \in \Omega$ as $x \rightarrow \pm \infty$;

(iv) there exist a function $\eta_{0} \in L^{\infty}(\Omega)_{+}$and $\delta_{0}>0$ such that

$$
\begin{aligned}
& \eta_{0}(z) \leqslant \hat{\lambda}_{m} \text { a.e. in } \Omega, \eta_{0} \neq \hat{\lambda}_{m}, \\
& \limsup _{x \rightarrow 0} \frac{2 F(z, x)}{x^{2}} \leqslant \eta_{0}(z) \text { uniformly for a.a. } z \in \Omega, \\
& 0 \leqslant F(z, x) \text { for a.a. } z \in \Omega \text {, all }|x| \leqslant \delta_{0} .
\end{aligned}
$$


Remark 6 . Note that in this case the perturbed monotonicity condition $H_{5}(i i)$ is in the opposite direction than before.

Example 5. The following function satisfies hypotheses $H_{5}$. As before, for simplicity no $z$-dependence is assumed:

$$
f(x)= \begin{cases}\eta_{0} x & \text { if }|x| \leqslant 1 \\ \lambda x-\left(\lambda-\eta_{0}\right) & \text { if } 1<|x|\end{cases}
$$

with $\hat{\lambda}_{k}<\lambda<0<\eta_{0}<\hat{\lambda}_{m}$.

As before (see Section [6), we assume that $0 \in \sigma(-\Delta+\beta)$ and this spectrum has a negative part. We set

$$
E=E(0), \hat{H}=\overline{\bigoplus_{i \geqslant m} E\left(\hat{\lambda}_{i}\right)} \text { and } V=E \oplus \hat{H} .
$$

Evidently $V$ is infinite dimensional.

Let $\varphi$ be the energy functional of the problem and $\psi=\left.\varphi\right|_{V}$. Evidently $\psi \in$ $C^{1}(V)$.

Proposition 24. If hypotheses $H_{0}$ and $H_{5}$ hold, then the functional $\psi$ satisfies the C-condition.

Proof. We do the proof for the case when the limit in $H_{5}(i i i)$ is $+\infty$. The proof is similar if the limit is $-\infty$.

So, let $\left\{y_{n}\right\}_{n \geqslant 1} \subseteq V$ be such that

$$
\begin{gathered}
\left|\psi\left(y_{n}\right)\right| \leqslant M_{9} \text { for some } M_{9}>0, \text { all } n \geqslant 1, \\
\left(1+\left\|y_{n}\right\|\right) \psi^{\prime}\left(y_{n}\right) \rightarrow 0 \text { in } V^{*} \text { as } n \rightarrow \infty .
\end{gathered}
$$

Claim 2. The sequence $\left\{y_{n}\right\}_{n} \geqslant 1 \subseteq H^{1}(\Omega)$ is bounded.

Arguing indirectly, we assume that $\left\|y_{n}\right\| \rightarrow \infty$. Let $w_{n}=\frac{y_{n}}{\left\|y_{n}\right\|}, n \geqslant 1$. Then $\left\|w_{n}\right\|=1$ for all $n \geqslant 1$, and so we may assume that

$$
w_{n} \stackrel{w}{\longrightarrow} w \text { in } H^{1}(\Omega) \text { and } w_{n} \rightarrow w \text { in } L^{2 s^{\prime}}(\Omega) .
$$

From (109) we have

$$
\begin{array}{r}
\left|\left\langle A\left(y_{n}\right), h\right\rangle+\int_{\Omega} \beta(z) y_{n} h d z-\int_{\Omega} f\left(z, y_{n}\right) h d z\right| \leqslant \frac{\varepsilon_{n}\|h\|}{1+\left\|y_{n}\right\|}, \\
\text { for all } h \in Y \text { with } \varepsilon_{n} \rightarrow 0^{+} .
\end{array}
$$

We multiply (111) with $\frac{1}{\left\|y_{n}\right\|}$, and choose $h=w_{n}-w \in H^{1}(\Omega)$. Then we have

$$
\begin{aligned}
& \left|\left\langle A\left(w_{n}\right), w_{n}-w\right\rangle+\int_{\Omega} \beta(z) w_{n}\left(w_{n}-w\right) d z-\int_{\Omega} \frac{f\left(z, y_{n}\right)}{\left\|y_{n}\right\|}\left(w_{n}-w\right) d z\right| \\
& \leqslant \frac{\varepsilon_{n}}{1+\left\|y_{n}\right\|}\left\|w_{n}-w\right\| \text { for all } n \geqslant 1, \\
\Rightarrow \quad & \lim _{n \rightarrow \infty}\left\langle A\left(w_{n}\right), w_{n}-w\right\rangle=0(\text { see }(110)), \\
\Rightarrow \quad & \left\|D w_{n}\right\|_{2} \rightarrow\|D w\|_{2}, \\
\Rightarrow \quad & w_{n} \rightarrow w \text { in } H^{1}(\Omega) \text { (by the Kadec-Klee property of Hilbert spaces), } \\
\Rightarrow & \|w\|=1 .
\end{aligned}
$$


Let $\Omega_{0}=\{z \in \Omega: w(z) \neq 0\}$. Evidently $\left|\Omega_{0}\right|_{N}>0$ and we have

$$
\left|y_{n}(z)\right| \rightarrow+\infty \text { for all } z \in \Omega_{0} .
$$

Then hypothesis $H_{5}($ iii) implies that

$$
2 F\left(z, y_{n}(z)\right)-f\left(z, y_{n}(z)\right) y_{n}(z) \rightarrow+\infty \text { for all } z \in \Omega_{0} .
$$

Using $H_{5}(i i i)$ and Fatou's lemma, we have

$$
\int_{\Omega}\left[2 F\left(z, y_{n}\right)-f\left(z, y_{n}\right) y_{n}\right] d z \rightarrow+\infty
$$

On the other hand, from (108) we have

$$
-\tau\left(y_{n}\right)+\int_{\Omega} 2 F\left(z, y_{n}\right) d z \leqslant 2 M_{9} \text { for all } n \geqslant 1 .
$$

Also, if in (111) we choose $h=y_{n} \in H^{1}(\Omega)$, then

$$
\tau\left(y_{n}\right)-\int_{\Omega} f\left(z, y_{n}\right) y_{n} d z \leqslant \varepsilon_{n} \text { for all } n \geqslant 1 .
$$

Adding (113) and (114), we obtain

$$
\int_{\Omega}\left[2 F\left(z, y_{n}\right)-f\left(z, y_{n}\right) y_{n}\right] d z \leqslant M_{10} \text { for some } M_{10}>0, \text { all } n \geqslant 1 .
$$

Comparing (112) and (115), we reach a contradiction. This proves the claim.

By virtue of the claim, we may assume that

$$
y_{n} \stackrel{w}{\longrightarrow} y \text { in } H^{1}(\Omega) \text { and } y_{n} \rightarrow y \text { in } L^{2 s^{\prime}}(\Omega) .
$$

As before, if in (111) we choose $h=y_{n}-y \in H^{1}(\Omega)$, pass to the limit as $n \rightarrow \infty$ and use (116), then via the Kadec-Klee property of Hilbert spaces, we have

$$
\begin{aligned}
& y_{n} \rightarrow y \text { in } H^{1}(\Omega), \\
\Rightarrow \quad & \psi \text { satisfies the } C \text {-condition. }
\end{aligned}
$$

We consider the following orthogonal direct sum decomposition of $H^{1}(\Omega)$ :

$$
H^{1}(\Omega)=\bar{H} \oplus V,
$$

where $\bar{H}=\bigoplus_{i=1}^{k} E\left(\hat{\lambda}_{i}\right)$ and $V=E \oplus \hat{H}$ (recall $E=E(0), \hat{H}=\overline{\bigoplus_{i \geqslant m} E\left(\hat{\lambda}_{i}\right)}$ ).

As we already mentioned, the reduction method will occur on the infinite dimensional space $V$.

Proposition 25. If hypotheses $H_{0}$ and $H_{5}$ hold, then there exists a continuous map $\tilde{\gamma}: V \rightarrow \bar{H}$ such that

$$
\varphi(v+\tilde{\gamma}(v))=\sup [\varphi(v+\bar{u}): \bar{u} \in \bar{H}] .
$$

Proof. The proof is similar to the proof of Proposition 10.

We fix $v \in V$ and consider the functional $\varphi_{v}: H^{1}(\Omega) \rightarrow \mathbb{R}$ defined by

$$
\varphi_{v}(u)=\varphi(v+u) \text { for all } u \in H^{1}(\Omega) .
$$

Let $i: \bar{H} \rightarrow H^{1}(\Omega)$ be the inclusion map and let $\bar{\varphi}_{v}: \bar{H} \rightarrow \mathbb{R}$ be defined by

$$
\bar{\varphi}_{v}=\varphi_{v} \circ i \text {. }
$$


The chain rule implies that

$$
\bar{\varphi}_{v}^{\prime}(\bar{u})=p_{\bar{H}^{*}} \varphi_{v}^{\prime}(\bar{u}) \text { for all } \bar{u} \in \bar{H},
$$

with $p_{\bar{H}^{*}}$ being the orthogonal projection on $\bar{H}^{*}$.

Let $\bar{u}_{1}, \bar{u}_{2} \in \bar{H}$. We have

$$
\begin{aligned}
& \left\langle\bar{\varphi}_{v}^{\prime}\left(\bar{u}_{1}\right)-\bar{\varphi}_{v}^{\prime}\left(\bar{u}_{2}\right), \bar{u}_{1}-\bar{u}_{2}\right\rangle_{\bar{H}} \\
= & \left\langle\varphi^{\prime}\left(v+\bar{u}_{1}\right)-\varphi^{\prime}\left(v+\bar{u}_{2}\right), \bar{u}_{1}-\bar{u}_{2}\right\rangle(\text { see }(\underline{117})) \\
= & \tau\left(\bar{u}_{1}-\bar{u}_{2}\right)-\int_{\Omega}\left[f\left(z, v+\bar{u}_{1}\right)-f\left(z, v+\bar{u}_{2}\right)\right]\left(\bar{u}_{1}-\bar{u}_{2}\right) d z \\
\leqslant & \tau\left(\bar{u}_{1}-\bar{u}_{2}\right)-\int_{\Omega} \eta(z)\left(\bar{u}_{1}-\bar{u}_{2}\right)^{2} d z\left(\text { see hypothesis } H_{5}(i i)\right) \\
\leqslant & -\xi_{2}\left\|\bar{u}_{1}-\bar{u}_{2}\right\|^{2}(\text { see Proposition } \underline{3}(a)), \\
\Rightarrow & \bar{u} \longmapsto-\bar{\varphi}_{v}(\bar{u})-\frac{\xi_{2}}{2}\|\bar{u}\|^{2}=\psi_{v}(\bar{u}) \text { is convex on } \bar{H} .
\end{aligned}
$$

Note that for all $\bar{u} \in \bar{H}$, we have

$$
\begin{aligned}
& \left\langle-\bar{\varphi}_{v}^{\prime}(\bar{u}), \bar{u}\right\rangle_{\bar{H}} \\
& =\left\langle-\bar{\varphi}_{v}^{\prime}(\bar{u})+\bar{\varphi}_{v}^{\prime}(0), \bar{u}\right\rangle_{\bar{H}}-\left\langle\bar{\varphi}_{v}^{\prime}(0), \bar{u}\right\rangle_{\bar{H}} \\
& \geqslant \xi_{2}\|\bar{u}\|^{2}-c_{8}\|\bar{u}\| \text { for some } c_{8}>0(\text { see }(\underline{118}) \text { ), } \\
& \quad \Rightarrow \bar{u} \longmapsto-\bar{\varphi}_{v}^{\prime}(\bar{u}) \text { is coercive. }
\end{aligned}
$$

Then from (118) and (120), it follows that $\bar{u} \longmapsto-\bar{\varphi}_{v}^{\prime}(\bar{u})$ is surjective (see, for example, Papageorgiou \& Kyritsi [26, p. 172]). So, we can find $\bar{u}_{0} \in \bar{H}$ such that

$$
\bar{\varphi}_{v}^{\prime}\left(\bar{u}_{0}\right)=0
$$

$\Rightarrow \bar{u}_{0}$ is the unique maximizer of the strictly concave functional $\bar{u} \longmapsto \bar{\varphi}_{v}(\bar{u})($ see (118) $)$.

So, we can define the map $\tilde{\gamma}: V \rightarrow \bar{H}$ by

$$
\tilde{\gamma}(v)=\bar{u}_{0}
$$

We have

$$
\bar{\varphi}_{v}^{\prime}(\tilde{\gamma}(v))=0 \text { and } \bar{\varphi}(\tilde{\gamma}(v))=\max [\varphi(v+\bar{u}): \bar{u} \in \bar{H}] .
$$

Claim 3. $\tilde{\gamma}: V \rightarrow \bar{H}$ is continuous.

Let $v_{n} \rightarrow v$ in $V$. From (121), we have

$$
\begin{aligned}
& \bar{\varphi}_{v_{n}}^{\prime}\left(\tilde{\gamma}\left(v_{n}\right)\right)=0 \text { for all } n \geqslant 1, \\
\Rightarrow \quad & \left\langle\bar{\varphi}_{v_{n}}\left(\tilde{\gamma}\left(v_{n}\right)\right), \tilde{\gamma}\left(v_{n}\right)\right\rangle_{\bar{H}}=0 \text { for all } n \geqslant 1,
\end{aligned}
$$$$
\Rightarrow\left\langle-\bar{\varphi}_{v_{n}}\left(\tilde{\gamma}\left(v_{n}\right)\right)+\bar{\varphi}_{v_{n}}^{\prime}(0), \tilde{\gamma}\left(v_{n}\right)\right\rangle_{\bar{H}}-\left\langle\bar{\varphi}_{v_{n}}^{\prime}(0), \tilde{\gamma}\left(v_{n}\right)\right\rangle_{\bar{H}}=0 \text { for all } n \geqslant 1 \text {, }
$$$$
\Rightarrow \xi_{2}\left\|\tilde{\gamma}\left(v_{n}\right)\right\|^{2} \leqslant c_{8}\left\|\tilde{\gamma}\left(v_{n}\right)\right\| \text { for all } n \geqslant 1 \text { (see (119)), }
$$

$\Rightarrow\left\{\tilde{\gamma}\left(v_{n}\right)\right\}_{n \geqslant 1} \subseteq \bar{H}$ is bounded.

Since $\bar{H}$ is finite dimensional, by passing to a suitable subsequence if necessary, we have

$$
\begin{aligned}
& \tilde{\gamma}\left(v_{n}\right) \rightarrow \bar{u}_{0} \text { in } \bar{H}, \\
\Rightarrow \quad & \varphi\left(v_{n}+\tilde{\gamma}\left(v_{n}\right)\right) \rightarrow \varphi\left(v_{n}+\bar{u}_{0}\right) .
\end{aligned}
$$


From (121) we have

$$
\begin{aligned}
& \varphi\left(v_{n}+\bar{u}\right) \leqslant \varphi\left(v_{n}+\tilde{\gamma}\left(v_{n}\right)\right) \text { for all } \bar{u} \in \bar{H}, \text { all } n \geqslant 1, \\
\Rightarrow & \varphi(v+\bar{u}) \leqslant \varphi\left(v+\bar{u}_{0}\right) \text { for all } \bar{u} \in \bar{H}, \\
\Rightarrow & \bar{u}_{0}=\tilde{\gamma}(v), \\
\Rightarrow & \tilde{\gamma} \text { is continuous. }
\end{aligned}
$$

We introduce $\xi(v)=\varphi(v+\tilde{\gamma}(v))$ for all $v \in V$. Reasoning as in the proof of Proposition 10, we obtain the following proposition.

Proposition 26. If hypotheses $H_{0}$ and $H_{5}$ hold, then $\xi \in C^{1}(V)$.

Recall that we are assuming that

$$
\sigma(-\Delta+\beta I) \cap(-\infty, 0) \neq \emptyset \text { and } 0 \in \sigma(-\Delta+\beta I) .
$$

We can now have our last multiplicity theorem.

Theorem 27. If hypotheses $H_{0}$ and $H_{5}$ hold, then problem (11) has at least two nontrivial solutions,

$$
u_{0}, \hat{u} \in C^{1}(\bar{\Omega}) .
$$

Proof. Hypotheses $H_{5}(i),(i i)$ imply that we can find $c_{9}>0$ such that

$$
F(z, x) \leqslant c_{9} \text { for a.a. } z \in \Omega \text {, all } x \in \mathbb{R} .
$$

Let $v \in V$. Then we have

$$
\begin{aligned}
\psi(v)=\varphi(v) & =\frac{1}{2} \tau(v)-\int_{\Omega} F(z, v) d z\left(\text { since } \psi=\left.\varphi\right|_{V}\right) \\
& \geqslant-\int_{\Omega} F(z, v) d z(\text { since } v \in V=E \oplus \hat{H}) \\
& \geqslant-c_{9}|\Omega|_{N}(\text { see }(\underline{122})), \\
& \Rightarrow \psi \text { is bounded below. }
\end{aligned}
$$

From Proposition 24 we know that $\psi$ satisfies the $C$-condition. This fact and (123) imply that $\psi$ is coercive (see Papageorgiou \& Kyristi [26, p. 272]).

Claim 4. $\xi$ satisfies the $C$-condition.

Let $\left\{v_{n}\right\}_{n \geqslant 1} \subseteq V$ such that

$$
\begin{aligned}
& \left|\xi\left(v_{n}\right)\right| \leqslant M_{11} \text { for some } M_{11}>0, \text { all } n \geqslant 1, \\
& \text { and }\left(1+\left\|v_{n}\right\|\right) \xi^{\prime}\left(v_{n}\right) \rightarrow 0 \text { in } V^{*} \text { as } n \rightarrow \infty .
\end{aligned}
$$

By definition we have

$$
\begin{aligned}
& \xi(v)=\varphi(v+\tilde{\gamma}(v)) \geqslant \varphi(v)=\psi(v) \text { for all } v \in V, \\
\Rightarrow \quad & \xi(\cdot) \text { is coercive (since } \psi \text { is coercive). }
\end{aligned}
$$

From this fact and (124) we infer that $\left\{v_{n}\right\}_{n \geqslant 1} \subseteq V$ is bounded. So, we may assume that

$$
v_{n} \stackrel{w}{\longrightarrow} v \text { in } H^{1}(\Omega) \text { and } v_{n} \rightarrow v \text { in } L^{2 s^{\prime}}(\Omega) .
$$


From (125) we have

$$
\begin{array}{r}
\left|\left\langle A\left(v_{n}\right), h\right\rangle+\int_{\Omega} \beta(z) v_{n} h d z-\int_{\Omega} f\left(z, v_{n}\right) h d z\right| \leqslant \frac{\varepsilon_{n}\|h\|}{1+\left\|v_{n}\right\|}, \\
\text { for all } v \in H^{1}(\Omega) \text { with } \varepsilon_{n} \rightarrow 0^{+} .
\end{array}
$$

In (127) we choose $h=v_{n}-v \in H^{1}(\Omega)$, pass to the limit as $n \rightarrow \infty$ and use (126). Then

$$
\begin{aligned}
& \lim _{n \rightarrow \infty}\left\langle A\left(v_{n}\right), v_{n}-v\right\rangle=0, \\
\Rightarrow \quad & \left\|D v_{n}\right\|_{2} \rightarrow\|D v\|_{2}, \\
\Rightarrow \quad & \left.v_{n} \rightarrow v \text { in } H^{1}(\Omega) \text { (by the Kadec-Klee property; see (126) }\right) .
\end{aligned}
$$

This proves Claim 4

Claim 5. $\xi^{\prime}(v)=p_{V^{*}} \varphi^{\prime}(v+\tilde{\gamma}(v))$ for all $v \in V\left(p_{V^{*}}\right.$ being the orthogonal projection of $H^{1}(\Omega)^{*}$ onto $\left.V^{*}\right)$.

For every $v, h \in V$, we have

$$
\begin{aligned}
&\left\langle\xi^{\prime}(v), h\right\rangle_{V}= \lim _{t \rightarrow 0} \frac{\xi(v+t h)-\xi(v)}{t} \\
&= \lim _{t \rightarrow 0} \frac{\varphi(v+t h+\tilde{\gamma}(v+t h))-\varphi(v+\tilde{\gamma}(v))}{t} \\
& \leqslant \lim _{t \rightarrow 0} \frac{\varphi(v+t h+\tilde{\gamma}(v+t h))-\varphi(v+\tilde{\gamma}(v+t h))}{t} \\
&=\left\langle\varphi^{\prime}(v+\tilde{\gamma}(v)), i_{0}(h)\right\rangle \\
&\left(\operatorname{since} \varphi \in C^{1}\left(H^{1}(\Omega)\right),\right. \\
&\left.\quad \text { with } i_{0}: V \rightarrow H^{1}(\Omega) \text { being the inclusion map }\right) \\
&=\left\langle p_{V^{*}} \varphi^{\prime}(v+\tilde{\gamma}(v)), h\right\rangle \text { for all } h \in V, \\
& \Rightarrow \xi^{\prime}(v)=p_{V^{*}} \varphi^{\prime}(v+\tilde{\gamma}(v)) .
\end{aligned}
$$

This proves Claim 5 .

Claim 6. $\xi$ has a local linking with respect to $V=E \oplus \hat{H}$.

Hypotheses $H_{5}(i),(i v)$ imply that given $\varepsilon>0$ and $r>2$, we can find $c_{10}=$ $c_{10}(\varepsilon, r)>0$ such that

$$
F(z, x) \leqslant \frac{1}{2}\left(\eta_{0}(z)+\varepsilon\right) x^{2}+c_{10}|x|^{r} \text { for a.a. } z \in \Omega, \text { all } x \in \mathbb{R} .
$$

Let $\hat{u} \in \hat{H}$. We have

$$
\begin{aligned}
\xi(\hat{u}) & =\varphi(\hat{u}+\tilde{\gamma}(\hat{u})) \\
& \geqslant \varphi(\hat{u}) \\
& =\frac{1}{2} \tau(\hat{u})-\int_{\Omega} F(z, \hat{u}) d z \\
& \geqslant \frac{1}{2}\left[\tau(\hat{u})-\int_{\Omega} \eta_{0}(z) u^{2} d z\right]-\frac{\varepsilon}{2}\|\hat{u}\|^{2}-c_{11}\|\hat{u}\|^{r} \text { for some } c_{11}>0 \\
& \geqslant \frac{\xi_{1}-\varepsilon}{2}\|\hat{u}\|^{2}-c_{11}\|\hat{u}\|^{r}(\text { see Proposition } 3(a)) .
\end{aligned}
$$


Choosing $\varepsilon \in\left(0, \xi_{1}\right)$ and since $r>2$, we can find $\varrho_{1} \in(0,1)$ small such that

$$
\xi(\hat{u}) \geqslant 0 \text { for all } \hat{u} \in \hat{H} \text { with }\|\hat{u}\| \leqslant \varrho_{1} .
$$

The space $Y=\bar{H} \oplus E$ is finite dimensional, and so all norms are equivalent. Therefore, we can find $\delta_{1}>0$ such that

$$
y \in Y \text { with }\|y\| \leqslant \delta_{1} \Rightarrow|y(z)| \leqslant \delta_{0} \text { for all } z \in \bar{\Omega} \text { (recall } Y \subseteq C^{1}(\bar{\Omega}) \text { ). }
$$

Here $\delta_{0}>0$ is as postulated by hypothesis $H_{5}(i v)$. Since $\tilde{\gamma}$ is continuous (see Proposition 25), we can find $\vartheta=\vartheta\left(\delta_{1}\right)>0$ such that

$$
e \in E \text { with }\|e\| \leqslant \vartheta \Rightarrow\|e+\tilde{\gamma}(e)\| \leqslant \delta_{1}(\text { recall } \tilde{\gamma}(0)=0) .
$$

From (130) and (131) it follows that if $e \in E$ with $\|e\| \leqslant \vartheta$, then

$$
\begin{aligned}
\xi(e)= & \varphi(e+\tilde{\gamma}(e)) \\
= & \frac{1}{2} \tau(e+\tilde{\gamma}(e))-\int_{\Omega} F(z, e+\tilde{\gamma}(e)) d z \\
\leqslant & -\int_{\Omega} F(z, e+\tilde{\gamma}(e)) d z(\text { since } e+\tilde{\gamma}(e) \in E \oplus \bar{H}=Y) \\
\leqslant & \left.0 \text { (see (131) and } H_{5}(i v)\right), \\
& \Rightarrow \xi(e) \leqslant 0 \text { for all } e \in E \text { with }\|e\| \leqslant \delta_{1} .
\end{aligned}
$$

If $\varrho=\min \left\{\varrho_{1}, \delta_{1}\right\}$, then from (129) and (132), we infer that $\xi$ has a local linking with respect to $V=E \oplus \hat{H}$.

This proves Claim 6 .

Note that $\xi(0)=0$, and so $\inf _{V} \xi \leqslant 0$. If $\inf _{V} \xi=0$, then from (132) we see that for all $e \in E$ with $0<\|e\| \leqslant \delta_{1}$, we have $\xi(e)=0=\inf _{V} \xi$. So, from Claim 5 we have

$$
\begin{aligned}
& 0=\xi^{\prime}(e)=p_{V^{*}} \varphi^{\prime}(e+\tilde{\gamma}(e)), \\
\Rightarrow \quad & e+\tilde{\gamma}(e)=\hat{u} \text { for all } e \in E \text { with } 0<\|e\| \leqslant \delta_{1} \text { is a solution of (1). }
\end{aligned}
$$

If $\inf _{V} \xi<0$, then we can apply Proposition 4 and produce $\hat{u}_{0}$ and $\tilde{u}$, two nontrivial critical points of $\xi$. Then

$$
u_{0}=\hat{u}_{0}+\tilde{\gamma}\left(\hat{u}_{0}\right) \text { and } \hat{u}=\tilde{u}+\tilde{\gamma}(\tilde{u})
$$

are two nontrivial solutions of problem (11). Regularity theory (see Wang 34) implies $u_{0}, \hat{u} \in C^{1}(\bar{\Omega})$.

\section{REFERENCES}

[1] Sergiu Aizicovici, Nikolaos S. Papageorgiou, and Vasile Staicu, Degree theory for operators of monotone type and nonlinear elliptic equations with inequality constraints, Mem. Amer. Math. Soc. 196 (2008), no. 915, vi+70, DOI 10.1090/memo/0915. MR2459421(2010c:47211)

[2] Herbert Amann, Saddle points and multiple solutions of differential equations, Math. Z. 169 (1979), no. 2, 127-166, DOI 10.1007/BF01215273. MR550724 (80j:47078)

[3] Thomas Bartsch, Critical point theory on partially ordered Hilbert spaces, J. Funct. Anal. 186 (2001), no. 1, 117-152, DOI 10.1006/jfan.2001.3789. MR1863294 (2002i:58011)

[4] Thomas Bartsch and Zhi-Qiang Wang, On the existence of sign changing solutions for semilinear Dirichlet problems, Topol. Methods Nonlinear Anal. 7 (1996), no. 1, 115-131. MR:1422008 (97m:35076)

[5] Haïm Brezis and Louis Nirenberg, Remarks on finding critical points, Comm. Pure Appl. Math. 44 (1991), no. 8-9, 939-963, DOI 10.1002/cpa.3160440808. MR1127041(92i:58032) 
[6] Alfonso Castro, Jorge Cossio, Carlos Vélez, Existence of seven solutions for asymptotically linear Dirichlet problem without symmetries, Ann. Mat. Pura Appl. (4) 192 (2013), no. 4, 607-619. MR3081637

[7] Alfonso Castro and Alan C. Lazer, Critical point theory and the number of solutions of a nonlinear Dirichlet problem, Ann. Mat. Pura Appl. (4) 120 (1979), 113-137, DOI 10.1007/BF02411940. MR551063 (81d:58022)

[8] Kung-ching Chang, Infinite-dimensional Morse theory and multiple solution problems, Progress in Nonlinear Differential Equations and their Applications, 6, Birkhäuser Boston, Inc., Boston, MA, 1993. MR1196690 (94e:58023)

[9] James Dugundji, Topology, Allyn and Bacon Inc., Boston (1996). MR0193606

[10] Michael Filippakis and Nikolaos S. Papageorgiou, Multiple nontrivial solutions for resonant Neumann problems, Math. Nachr. 283 (2010), no. 7, 1000-1014, DOI 10.1002/mana.200710045. MR2677284 (2011g:35142)

[11] Leszek Gasiński and Nikolaos S. Papageorgiou, Nonlinear analysis, Series in Mathematical Analysis and Applications, vol. 9, Chapman \& Hall/CRC, Boca Raton, FL, 2006. MR2168068 (2006e:47001)

[12] Leszek Gasiński and Nikolaos S. Papageorgiou, Dirichlet problems with double resonance and an indefinite potential, Nonlinear Anal. 75 (2012), no. 12, 4560-4595, DOI 10.1016/j.na.2011.09.014. MR2927120

[13] Leszek Gasiński and Nikolaos S. Papageorgiou, Pairs of nontrivial solutions for resonant Neumann problems, J. Math. Anal. Appl. 398 (2013), no. 2, 649-663, DOI 10.1016/j.jmaa.2012.09.034. MR2990090

[14] Andrzej Granas and James Dugundji, Fixed point theory, Springer Monographs in Mathematics, Springer-Verlag, New York, 2003. MR.1987179 (2004d:58012)

[15] Helmut Hofer, Variational and topological methods in partially ordered Hilbert spaces, Math. Ann. 261 (1982), no. 4, 493-514, DOI 10.1007/BF01457453. MR682663 (84g:58030)

[16] Shouchuan $\mathrm{Hu}$ and Nikolas S. Papageorgiou, Double resonance for Dirichlet problems with unbounded indefinite potential and combined nonlinearities, Commun. Pure Appl. Anal. 11 (2012), no. 5, 2005-2021, DOI 10.3934/cpaa.2012.11.2005. MR2911122

[17] Sophia Th. Kyritsi and Nikolaos S. Papageorgiou, Multiple solutions for superlinear Dirichlet problems with an indefinite potential, Ann. Mat. Pura Appl. (4) 192 (2013), no. 2, 297-315, DOI 10.1007/s10231-011-0224-z. MR3035141

[18] Shibo Liu, Remarks on multiple solutions for elliptic resonant problems, J. Math. Anal. Appl. 336 (2007), no. 1, 498-505, DOI 10.1016/j.jmaa.2007.01.051. MR2348521(2008f:35126)

[19] Jia Quan Liu and Shu Jie Li, An existence theorem for multiple critical points and its application (Chinese), Kexue Tongbao (Chinese) 29 (1984), no. 17, 1025-1027. MR.802575

[20] Shibo Liu and Shujie Li, Critical groups at infinity, saddle point reduction and elliptic resonant problems, Commun. Contemp. Math. 5 (2003), no. 5, 761-773, DOI 10.1142/S0219199703001129. MR2017717(2004i:58013)

[21] C. R. F. Maunder, Algebraic topology, Dover Publications, Inc., Mineola, NY, 1996. Reprint of the 1980 edition. MR,1402473 (97c:55001)

[22] Dumitru Motreanu, Viorica V. Motreanu, and Nikolaos S. Papageorgiou, On resonant Neumann problems, Math. Ann. 354 (2012), no. 3, 1117-1145, DOI 10.1007/s00208-011-0763-z. MR2983082

[23] Dumitru Motreanu and Nikolaos S. Papageorgiou, Multiple solutions for nonlinear Neumann problems driven by a nonhomogeneous differential operator, Proc. Amer. Math. Soc. 139 (2011), no. 10, 3527-3535, DOI 10.1090/S0002-9939-2011-10884-0. MR2813384(2012c:35166)

[24] Donal O'Regan, Nikolaos S. Papageorgiou, and George Smyrlis, Neumann problems with double resonance, Topol. Methods Nonlinear Anal. 39 (2012), no. 1, 151-173. MR2952308

[25] Nikolaos S. Papageorgiou and Sophia Th. Kyritsi-Yiallourou, Handbook of applied analysis, Advances in Mechanics and Mathematics, vol. 19, Springer, New York, 2009. MR 2527754 (2010g:49001)

[26] Nikolaos S. Papageorgiou and Francesca Papalini, Seven solutions with sign information for sublinear equations with unbounded and indefinite potential and no symmetries, Israel J. Math., in press.

[27] Nikolaos S. Papageorgiou and Vicenţiu D. Rădulescu, Semilinear Neumann problems with indefinite and unbounded potential and crossing nonlinearity, Recent trends in nonlinear 
partial differential equations. II. Stationary problems, Contemp. Math., vol. 595, Amer. Math. Soc., Providence, RI, 2013, pp. 293-315, DOI 10.1090/conm/595/11801. MR3156380

[28] Nikolaos S. Papageorgiou and George Smyrlis, On a class of parametric Neumann problems with indefinite and unbounded potential, Forum Math. DOI: 10.1515/forum-2012-0042.

[29] Patrizia Pucci and James Serrin, The maximum principle, Progress in Nonlinear Differential Equations and their Applications, 73, Birkhäuser Verlag, Basel, 2007. MR2356201 (2008m:35001)

[30] Chun-Lei Tang, Multiple solutions of Neumann problem for elliptic equations, Nonlinear Anal. 54 (2003), no. 4, 637-650, DOI 10.1016/S0362-546X(03)00091-9. MR1983440 (2004b:35121)

[31] Chun-Lei Tang and Xing-Ping Wu, Existence and multiplicity for solutions of Neumann problem for semilinear elliptic equations, J. Math. Anal. Appl. 288 (2003), no. 2, 660-670, DOI 10.1016/j.jmaa.2003.09.034. MR2020187 (2005a:35093)

[32] Klaus Thews, Nontrivial solutions of elliptic equations at resonance, Proc. Roy. Soc. Edinburgh Sect. A 85 (1980), no. 1-2, 119-129, DOI 10.1017/S0308210500011732. MR566069 (81c:35054)

[33] Juan Luis Vázquez, A strong maximum principle for some quasilinear elliptic equations, Appl. Math. Optim. 12 (1984), no. 3, 191-202, DOI 10.1007/BF01449041. MR768629 (86m:35018)

[34] Xu Jia Wang, Neumann problems of semilinear elliptic equations involving critical Sobolev exponents, J. Differential Equations 93 (1991), no. 2, 283-310, DOI 10.1016/00220396(91)90014-Z. MR.1125221 (92j:35072)

Department of Mathematics, Zografou Campus, National Technical University, Athens 15780, Greece

E-mail address: npapg@math.ntua.gr

Department of Mathematics, Faculty of Science, King Abdulaziz University, Jeddah, Saudi Arabia - and - Institute of Mathematics "Simion Stollow" of the Romanian AcAdemy, P.O. Box 1-764, 014700 Bucharest, Romania

E-mail address: vicentiu.radulescu@math.cnrs.fr 